\title{
Plebiszite in der Zustimmungsdiktatur - Die nationalsozialistischen Volksabstimmungen 1933, 1934 und 1938: das Beispiel Schleswig- Holstein
}

\section{Frank Omland}

Was tun Menschen, wenn sie in einer Diktatur wählen müssen? Warum und wozu inszeniert ein Regime Wahlen, wenn es den Menschen keine Wahl lassen will? Wie bewerten die Machthaber und deren verfolgte Gegner die Ergebnisse von unfreien Abstimmungen? Und lassen sich aus den Wahlergebnissen Rückschlüsse auf die Verfasstheit der Gesellschaft in einer Diktatur ziehen? ${ }^{1}$ Das sind Fragen, die in einem „Jahrbuch für direkte Demokratie“ fehl am Platze zu sein scheinen, denn Wahlen und Diktatur schließen sich per se aus und der Erkenntnisgewinn von unfreien Wahlen für die Erforschung der Praxis von Elementen der direkten Demokratie ist vordergründig eher gering. Wieso also ein Beitrag zu den nationalsozialistischen Volksabstimmungen in diesem Jahrbuch? Ein Teil der Antwort besteht darin, dass anfangs auch in der NS-Diktatur die Wahlberechtigten noch eine Wahl hatten. Da das Regime mit den Abstimmungen innen- und außenpolitische Legitimationsziele verband, konnten diese nur erreicht werden, indem zumindest noch 1933 und 1934 bestimmte Standards von „freien“ Wahlen eingehalten wurden. Erst in Folge der Konsolidierung der NS-Herrschaft traten die diktatorischen Züge des Regimes so sehr in den Vordergrund, dass eine angebliche Handlungsfreiheit der Wahlberechtigten bei den Abstimmungen in der Praxis vollständig ad absurdum geführt wurde und endgültig von Wahlen ohne Wahl gesprochen werden kann.

Damit stellten die Abstimmungen in der Diktatur auch immer ein Problem für die Herrschenden dar, denn die Nationalsozialisten glaubten an die Entstehung der „Volksgemeinschaft“, an die Einheit von Führer und Geführten. Dies bedeutete zwangsläufig, dass alle Wahlberechtigten mehr oder minder freiwillig für das Regime stimmen würden, was im Umkehrschluss wiederum als Beleg für die Werdung der Volksgemeinschaft gewertet werden konnte. Wenn ,,Volk und Führer eins!“‘2 waren, konnte es innerhalb dieser Logik gar nicht zu Abweichungen kommen. Dementsprechend irritierend und im Kern tatsächlich nicht verstehbar war für die Machthaber oppositionelles und widerständiges Handeln bei den Volksabstimmungen und Reichstagswahlen.

Es stellt sich damit die provozierende Frage, ob Abstimmungen in Diktaturen nicht zwangsläufig immer mehr zur De-Legitimierung denn zur Legitimierung des

1 Vgl. dazu R. Jessen/H. Richter, Internationale historische Konferenz. Wahlen in den Diktaturen des 20. Jahrhunderts/Elections under 20th Century Dictatorships. Historisches Seminar der Universität zu Köln, 7.5.-9.5.2009 [Unv. 2009]. - Ein englischsprachiger Tagungsband erscheint 2010.

2 Kieler Zeitung, 1.4.1936. 
Regimes führen müssen: ${ }^{3}$ Entweder sind es die unglaubwürdig hohen Zustimmungsergebnisse, die weder der eigenen Anhängerschaft noch den Gegnern vermittelt werden können - so geschehen im Nationalsozialismus bei der Reichstagswahl 1936 - oder das Regime delegitimiert sich dadurch, dass es die hohe Ablehnungsquote, den Dissens sichtbar werden lässt - so bei der Volksabstimmung 1934. Die Frage ist, ob das faktische Scheitern von Diktaturen am System von Wahlen und Abstimmungen also per se im Positiven als Beleg für plebiszitäre Elemente gewertet werden könnte?

\section{Einführung}

Die zweitgrößte nationalsozialistische Zeitung Schleswig-Holsteins, die Nordische Rundschau in Kiel, ${ }^{4}$ titelte in ihrer Wochenendausgabe vom 11./12. August 1934 mit der Frage „Hitler - Demokrat oder Diktator?“ Darin hieß es eingangs: „Demokratie hat keinen guten Ruf in Deutschland. [...] Es gibt eine Demokratie rein deutschen Ursprungs, aber auch eine aus französisch-jüdischem Geist. Diesen Unterschied muß man kennen. Die deutsche ist aber allein die echte Demokratie. [...] Wir haben alle Veranlassung, die zum Staats- und Volksuntergang führende, liberale Demokratie jüdisch-französischer Prägung scharf von dem deutsch-demokratischen Begriff germanischer Selbstverwaltung unter autoritärer aus dem Volk geborener Führung zu trennen. Das ist deshalb notwendig, damit wir den Unterschied zwischen Führung im Sinne Hitlers und Diktatur begreifen lernen. [...] Einen Diktator haben wir in Mussolini vor uns. [...] Und was Staat ist, bestimmt er allein. Das Volk hat zu parieren. [...] Seine Diktatur beruht allein auf der Macht der Bajonette, über die er verfügt. Daß wir in Deutschland keine Diktatur haben, danken wir allein dem Führer Adolf Hitler. [...] Und wiederum zeigt sich der Führer als Volksmann, der den Staat und seine Führung im Einklang mit der Gesamtheit des Volkes wissen will. So ruft er sein Volk nun zur Wahl. Wieder soll es sprechen und seine ungeschminkte Meinung sagen, ungehemmt von jeder Beeinflussung durch Staat und Partei. Nur seine Leistungen und Taten sollen für ihn sprechen. " 5

Dieser Zeitungsartikel im Zuge der Propaganda zur Volksabstimmung im August 1934 beinhaltet viele der zentralen Topoi in der Argumentation der Nationalsozialisten: die antisemitisch unterlegte Diffamierung der liberalen Demokratie, die Abgrenzung zu anderen Diktaturen, ${ }^{6}$ die behauptete Entstehung der Volksgemein-

3 Vgl. dazu W. Patzelt, Elections in Modern Dictatorships: An Analytical Framework. [Unv. 2009] Der Politologe Werner Patzelt vertrat auf der Internationalen Konferenz (Fn. 1) die These, dass auf lange Sicht betrachtet, Wahlen und Abstimmungen zur Destabilisierung der Diktatur beitragen würden.

4 Daten nach M. Oddey, „Unter Druck gesetzt“" Presse und Nationalsozialismus in SchleswigHolstein, 2006.

5 Nordische Rundschau 11./12.8.1934, „Hitler - Demokrat oder Diktator?“

6 Vgl. P. Corner, Fascist national unity and the importance of the appearance of unity, [Unv. 2009, Fn. 1] sowie E. Fimiani, Plebiscites in Fascist Italy and Nazi-Germany: comparative perspectives. [Unv. 2009, Fn. 1]. 
schaft, die Legitimierung der eigenen Herrschaft aufgrund von Volksabstimmungen und die Proklamation einer ,echten“ deutschen Demokratie, weil der „Führer“ die Beherrschten befragen lässt (obwohl er es eigentlich nicht bräuchte) und sich damit eben nicht als Diktator geriert, sondern als „,wahrer deutscher Demokrat“. Die NSPlebiszite dienten also zur Legitimierung der eigenen Herrschaft, doch gab es einen Widerspruch zwischen der von den Machthabern behaupteten Möglichkeit zur freien Meinungsäußerung bei den Volksabstimmungen und der sozialen Kontrolle durch Partei- und Volksgenossen bis hin zu Denunziationen, die zu Strafverfolgungen mit Hilfe der Sondergerichte führten. ${ }^{7}$ Das NS-Regime wollte, dass alle Volksgenossen

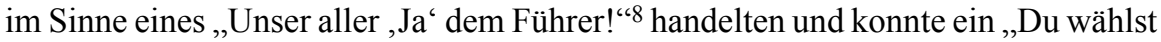
mi nich Hitler!“9 in diesem Kalkül nur noch als widerständiges, zu verfolgendes Verhalten werten. Dass sich trotzdem nicht wenige Wählerinnen und Wähler in Schleswig-Holstein vor bzw. im Wahllokal und mit Hilfe des Stimmzettels gegen das NS-Regime und seine Politik aussprachen, ist ein stark vernachlässigtes, wenn nicht sogar vergessenes Kapitel der Oppositions- und Widerstandsgeschichte des „Dritten Reiches“.

Im Folgenden werden thesenartig und zusammenfassend die Ergebnisse der regionalgeschichtlichen Forschungen für Schleswig-Holstein für die Volksabstimmungen vom 12. November 1933 und 19. August 1934 sowie die „Volksabstimmung und Wahl zum Großdeutschen Reichstag“" vom 10. April 1938 vorgestellt $^{10}$ und anschließend ein Ausblick darauf gegeben, was in Zukunft die historische Wahlforschung noch leisten sollte.

\section{Die Ausgangslage: Schleswig-Holstein als frühe Hochburg der NSDAP}

Die Nationalsozialisten waren in Schleswig-Holstein relativ früh und kontinuierlich bei demokratischen Wahlen erfolgreich, was für die Abstimmungen in der NS-Diktatur erst einmal hohe Zustimmungsergebnisse erwarten lassen sollte; dementsprechend ungewöhnlich erscheinen die aufgetretenen Abweichungen [Abb. 1]. ${ }^{11}$

7 Für Schleswig-Holstein sind knapp ein Dutzend Sondergerichtsverfahren für die Jahre 1933, 1934 und 1936 überliefert. Bei den meisten handelt es sich um Strafverfahren nach Denunziationen aus dem Alltagsumfeld, d.h. bei Gesprächen am Arbeitsplatz, in der Kneipe oder in Geschäften.

8 Kieler Neueste Nachrichten 12.11.1933, Titelschlagzeile.

9 Schleswig-Holsteinische Tageszeitung, Gauzeitung der NSDAP, 13.11.1933: „Zwei widerwärtige Zeitgenossen“ [Bericht über die Verhaftung zweier Männer vor einem Wahllokal in Itzehoe aufgrund von „,defätistischen“ Äußerungen].

10 Vgl. F. Omland, „Du wählst mi nich Hitler!“ Reichstagswahlen und Volksabstimmungen in Schleswig-Holstein 1933-1938, 2006. - Zur Region Altona und Hamburg siehe dens., „Auf Deine Stimme kommt es an!“ Die Reichstagswahl und Volksabstimmung am 12. November 1933 in Altona, in: Zeitschrift des Vereins für Hamburgische Geschichte 94 (2008), S. 57 ff.

11 Prozentuierung aller folgenden Ergebnisse auf Basis der Wahlberechtigten (siehe Exkurs). 


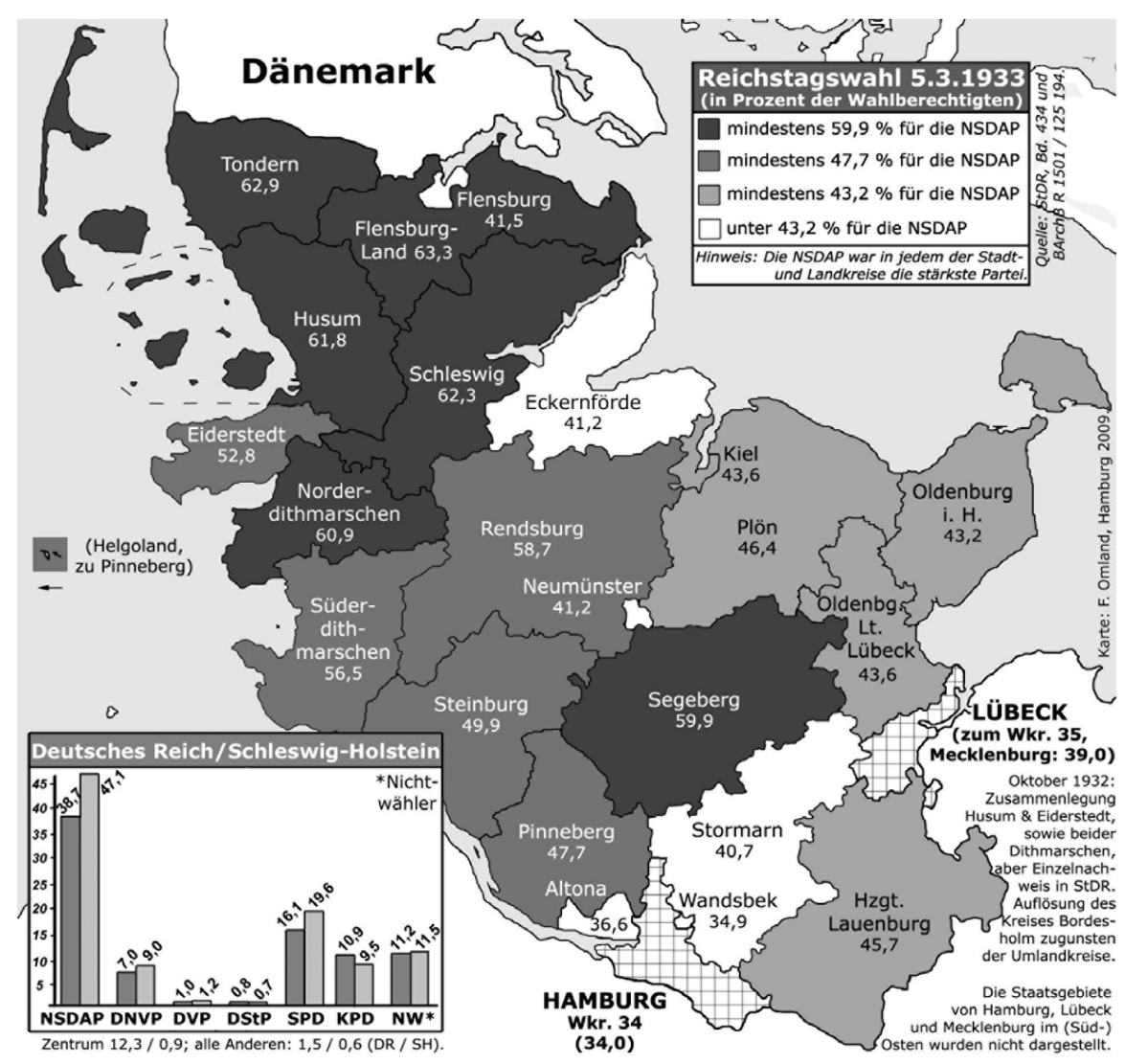

Der Wahlkreis 13 des Deutschen Reiches, d.h. die preußische Provinz SchleswigHolstein und der oldenburgische Landesteil Lübeck, wird für die Weimarer Republik landläufig als frühe und sehr erfolgreiche Hochburg der NSDAP beschrieben. Doch rangierten die völkisch-antisemitischen Zweckbündnisse und Vorläufer der Partei in Schleswig-Holstein bei den Reichstagswahlen vom Mai und Dezember $1924^{12}$ trotz einiger bemerkenswert hoher Ergebnisse in bestimmten Gemeinden ${ }^{13}$ reichsweit nur an 14. bzw. 18. Stelle aller Wahlkreise. ${ }^{14}$ Erst mit der Reichstagswahl

12 Deutsches Reich/Schleswig-Holstein, in Prozent der Wahlberechtigten: VSB, Mai 1924: 5,0/5,6; NSFB, Dez. 1924: 2,3/2,0.

13 Hohenwestedt/Kreis Rendsburg 20,3 \% der Wahlberechtigten; Mölln/Kreis Hzgt. Lauenburg 19,5\%; Kappeln/Kreis Schleswig 19,4\%. Vgl. im Detail die Tabelle 4 in: F. Omland, „Die Nordmark steht zu Hitler!“. Wählerwanderungen und Wählerherkunft der NSDAP 1924-1933, in: Informationen zur Schleswig-Holsteinischen Zeitgeschichte 48 (2007), S. 46.

14 Reichsweit siehe J.W. Falter, Hitlers Wähler, 1991, S. 155 und 160. - Für Schleswig-Holstein: Omland, Nordmark (Fn. 13), S. 16 ff., 42 u. 46 sowie F. Omland, „Gegen das System!““ Zum Aufstieg der NSDAP bei den Wahlen in Schleswig-Holstein 1924-1933, in: Informationen zur Schleswig-Holsteinischen Zeitgeschichte, Heft 50 (2008), S. 28 ff. - Vgl. auch 
1928 verschob sich die regionale Verteilung der Hochburgen der NSDAP nach Norden und Nordosten der Reichstagswahlkreise, und die Partei hatte ihre größten Erfolge in ,agrarischen Regionen mit evangelischer Bevölkerungsmehrheit und in überwiegend protestantischen Kleingemeinden ". ${ }^{15}$ In Schleswig-Holstein errang die NSDAP mit 3,0 Prozent der Wahlberechtigten (Deutsches Reich: 2,0 Prozent) Rang 7 von 35 Wahlkreisen. Doch dort, wo die Partei besonders umfangreiche Propaganda betrieb, in den Landkreisen und dörflichen Gemeinden der südwestlichen Küstenregionen (Kreis Norder- und Süderdithmarschen) deutete sich mit knapp 12 Prozent schon das Potential der Partei bei den nachfolgenden Wahlen an. ${ }^{16}$ Die nächste Etappe des Aufstiegs waren die preußischen Provinzial-Landtagswahlen im November 1929, wo die NSDAP mit etwa 7 Prozent der Wahlberechtigten das beste Ergebnis aller Provinzen im größten Land des Reiches verbuchen konnte. ${ }^{17}$ Bis 1933 sollte die schleswig-holsteinische NSDAP bei allen folgenden Wahlen diese führende Position nicht mehr abgeben und in der Regel über 10 Prozentpunkte mehr Stimmen der Wahlberechtigten für sich gewinnen als in den übrigen Regionen Preußens und des Deutschen Reiches. ${ }^{18}$ Erst bei der Reichstagswahl vom 5. März 1933 (47,1 zu 38,7 Prozent reichsweit) musste sie sich nach Frankfurt/Oder (49,0 Prozent), Pommern (48,6 Prozent), Liegnitz (48,3 Prozent), Osthannover (48,2 Prozent) und Ostpreußen (48,1 Prozent) „,nur“ mit Platz 6 aller Wahlkreise begnügen. Vor diesem Hintergrund war zu erwarten, dass die NSDAP auch in der Diktatur in ihren schleswig-holsteinischen Hochburgen besonders gut bei den Abstimmungen abschneiden würde; dass das hingegen nicht der Fall war, wird uns in der folgenden Analyse der Ereignisse noch beschäftigen.

\section{Gleichschaltung der Länder, Einparteienstaat, Plebiszite und Reichstagswahlen}

Nach der letzten pluralistischen Reichstagswahl vom 5. März 1933 sorgte das NSRegime durch das „Vorläufige Gesetz zur Gleichschaltung der Länder mit dem

R. Rietzler, „Kampf in der Nordmark“. Das Aufkommen des Nationalsozialismus in Schleswig-Holstein (1919-1928), 1982, S. $219 \mathrm{ff} ., 251 \mathrm{ff}$.

15 Falter, Hitlers Wähler (Fn. 14), S. 163 u. 160. - Vgl. den Kontrastgruppenvergleich bei J. Falter/T. Lindenberger/S. Schumann, Wahlen und Abstimmungen in der Weimarer Republik, 1986, S. 200. - Zur Bewertung der Propaganda und des Wahlkampfes 1929 siehe G. Paul, Aufstand der Bilder. Die NS-Propaganda vor 1933, 1990, S. 68.

16 Norderdithmarschen: 11,9\%; Süderdithmarschen: 12,1\%; die besten Ergebnisse errang die Partei in Gemeinden mit 4.000 bis 6.000 Einwohnern (Albersdorf: 32,1\%).

17 Eigene Berechnungen nach Statistisches Jahrbuch für den Freistaat Preußen, Bd. 26, 1930, S. 354 f., unter Hochrechnung der Wahlberechtigtenziffern für Preußen. Vgl. im Detail F. Omland, „Wie wähle ich?“ Die Provinziallandtagswahlen in Schleswig-Holstein 1921-1933, in: D. Kraack/K.-J. Lorenzen-Schmidt (Hrsg.), Brückenschläge aus der Vergangenheit. Festschrift für Peter Wulf zu seinem 70. Geburtstag, 2008, S. 282 ff.

18 Reichstagswahl 14. September 1930: 22,1 \% (Reich 14,9\%); Landtagswahl 24. April 1932: 43,3 \% (Preußen: 29,6\%); Reichstagswahl 31. Juli 1932: 44,3\% [= 51,0 der abgegebenen gültigen Stimmen] zu 31,1 \%; Reichstagswahl 6. November 1932: $38,4 \%$ zu 26,5 \%. 
Reich“19 sowie das „Zweite Gesetz zur Gleichschaltung der Länder“20 dafür, dass sich sämtliche Länderparlamente unter Ausschluss der KPD-Mandate analog zum Reichstagswahlergebnis umbilden mussten und damit die NSDAP in den Ländern zur stärksten Partei wurde. Das Verbot der Arbeiterparteien und „nackte Angst vor Repressalien, Einschüchterung und Verfolgung bis zur Inhaftierung und Mord auf der einen Seite, Kleinmut, Opportunismus und Beflissenheit auf der anderen“ führten dazu, dass sich alle bürgerlichen Parteien bis Ende Juni/Anfang Juli 1933 selbst auflösten. ${ }^{21}$ Die pseudolegale Monopolisierung der NSDAP zur einzigen Partei in Deutschland wurde am 14. Juli 1933 durch das „Gesetz gegen die Neubildung von Parteien" "22 besiegelt und das Verhältnis zwischen Partei und Staat am 1. Dezember 1933 mit dem „Gesetz zur Sicherung der Einheit von Partei und Staat“ scheinbar festgeschrieben, wobei dieses die Kompetenz- und Funktionsbegrenzungen zwischen beiden bewusst in der Schwebe ließ.23

In der Folge wurden die Länderparlamente ohne Neuwahlen im November 1933 aufgelöst und Ende Januar 1934 durch das „Gesetz über den Neuaufbau des Reiches“ Hoheitsrechte der Länder auf das Reich übertragen. Damit fanden in der NS-Zeit weder auf Länderebene noch in den Gemeinden Wahlen statt, ${ }^{24}$ so dass die Reichstagswahlen und die Volksabstimmungen auf Reichsebene die einzige Möglichkeit für die Wahlberechtigten darstellten, ihre Zustimmung bzw. ihre Opposition zum Einparteienstaat der NSDAP zu artikulieren. Die ersten Reichstagswahlen sowie eine Volksabstimmung (Legitimierung des Austritts aus dem Völkerbund) fanden am 12. November 1933 statt, es folgten eine Volksabstimmung am 19. August 1934 (Reichspräsidentschaft Hitlers), die Reichstagswahl am 29. März 1936 (Legitimierung des Einmarsches ins entmilitarisierte Rheinland), die „Volksabstimmung und Wahl zum Großdeutschen Reichstag" am 10. April 1938 (Legitimierung der Annexion Österreichs) sowie am 6. Dezember 1938 noch die Sudetendeutsche Ergänzungswahl zum Reichstag. ${ }^{25}$ Im Folgenden sollen die drei Plebiszite von 1933, 1934 und 1938 näher beleuchtet und bewertet werden.

19 Reichsgesetzblatt [RGB1.] 1933 I, S. 153, „Vorläufiges Gesetz zur Gleichschaltung der Länder“, 31.3.1933.

20 RGB1. 1933 I, S. 173, 7.4.1933, „Zweites Gesetz zur Gleichschaltung der Länder mit dem Reich“.

21 B.J. Wendt, Deutschland 1933-1945. Das Dritte Reich. Handbuch zur Geschichte, 1995, S. 111.

22 RGB1. 1993 I, S. 479, 14.7.1933, „Gesetz gegen die Neubildung von Parteien“.

23 RGB1. 1933 I, S. 1016, 1.12.1933, „Gesetz zur Sicherung der Einheit von Partei und Staat“. Vgl. zu den Auswirkungen: Wendt, Deutschland (Fn. 21), S. 117.

24 RGB1. 1934 I, S. 75, 30.1.1934 „Gesetz über den Neuaufbau des Reichs“.

25 Für eine Gesamtdarstellung der Wahlen in der Diktatur müssen ebenfalls die VertrauensräteWahlen in den Betrieben (April 1934 und 1935) sowie die Saarabstimmung vom 13. Januar 1935 einbezogen werden. Zusammen könnten so auf der Mikroebene Aussagen über die Verfasstheit der „Volksgemeinschaft“ und über die Auswirkungen von Nationalismus und Nationalgefühl - auch und gerade in der linken Arbeiterbewegung getroffen werden. 


\section{Legitimierung durch Einhaltung formaler Verfahren und Abläufe}

Um die Fiktion einer scheinbar freien „Abstimmung“ aufrecht erhalten zu können, knüpften die Nationalsozialisten zu Beginn ihrer Herrschaft stark an die in der Weimarer Republik geschaffenen formalen und juristischen Abläufe an. Durch die faktische Übernahme fast aller Regeln, die den Wahlberechtigten noch aus der Demokratie bekannt waren, versicherte sich das NS-Regime einer Legitimität, die es benötigte, um die diktatorischen Züge bei den Plebisziten und Wahlen für die Öffentlichkeit weniger offensichtlich werden zu lassen. Je scheinbar freier die Nationalsozialisten diese abliefen ließen, desto größer war für sie der zu erwartende politische Gewinn bei einer entsprechend hohen Zustimmungsquote durch die Wahlberechtigten.

In der Praxis blieben in der NS-Diktatur 1933 und 1934 sämtliche formalen Abläufe, alle Gesetze und Verwaltungsvorschriften der Weimarer Republik bestehen, ${ }^{26}$ und es wurde auch keine Wahlpflicht eingeführt. Lediglich das bisherige System von Volksbegehren und Volksentscheid, das faktisch die Wahlenthaltungen förderte, wurde durch ein neues Gesetz abgelöst: das „Gesetz über Volksabstimmung“", ${ }^{27}$ welches das Abstimmungsverfahren vereinfachte. ${ }^{28}$

Die Aufrechterhaltung möglichst vieler Formalien aus der Zeit der Weimarer Republik sollte sowohl gegenüber dem Ausland als auch im Inland die Vertrauens- und Glaubwürdigkeit des Regimes erhöhen und den Schein von Normalität bei den Wahlen und Abstimmungen herstellen. So konnten die Wahlberechtigten auf eine trügerische Sicherheit setzen, die von öffentlicher Auslage der Wählerverzeichnisse über die Anschreiben mit den Wahlbenachrichtigungen und Wahllokale mit Wahlvorständen, die die Abläufe überprüften, bis zu der geheimen Abstimmung in den Wahlkabinen reichte. Außerdem bestand nach der Ausstellung eines Stimmscheins die Möglichkeit, außerhalb des eigenen Stimmbezirks zur Wahl zu gehen (oder dies zu vermeiden). ${ }^{29}$ Die soziale Kontrolle der Stimmscheinwähler bereitete der NSDAP noch 1934 Kopfzerbrechen, denn diese standen unter dem generellen Verdacht, potentielle Nichtwähler zu sein. ${ }^{30}$ Die Höhe der Wahlbeteiligung sollte dem Regime noch 1933 und 1934 so wichtig sein, dass der von der Partei gewünschte

26 Vgl. C. Schwieger, Volksgesetzgebung in Deutschland. Der wissenschaftliche Umgang mit plebiszitärer Gesetzgebung auf Reichs- und Bundesebene in Weimarer Republik, Drittem Reich und Bundesrepublik Deutschland (1919-2002), 2005, S. 212 ff. (1938 erließ das Regime neue Vorschriften, die im Ablauf aber den ,alten“ ähnelten).

27 RGB1. 1933 I, S. 479, „Gesetz über Volksabstimmung“ vom 14. Juli 1933. - Vgl. zur Genese des Gesetzes Schwieger, Volksgesetzgebung (Fn. 26), S. $203 \mathrm{ff}$.

28 Zur Diskussion um seine Bedeutung vgl. O. Jung, Plebiszit und Diktatur: die Volksabstimmungen der Nationalsozialisten. Die Fälle „Austritt aus dem Völkerbund“ (1933), „Staatsoberhaupt“ (1934) und „Anschluß Österreichs“ (1938), 1995, S. 21, 31 ff.

29 Vgl. Omland, Du wählst (Fn. 10), S. 36.

30 Kreisarchiv Schleswig-Flensburg 9/26. NSDAP Ortsgruppe Schleswig, Posteingang beim Magistrat Schleswig am 6.11.1933. 
Ausschluss der jüdisch-gläubigen Wahlberechtigten ausblieb. ${ }^{31}$ Erst nach Konsolidierung der NS-Herrschaft und der fast 99-prozentigen Wahlbeteiligung setzten sich innerhalb des Machtapparats diejenigen durch, die auf Ausschluss der nicht zur „Volksgemeinschaft“ Gehörigen drängten. In der Folge der Verabschiedung der rassistischen Gesetze gegen als ,jüdisch“ definierte Bevölkerungsgruppen entzog man ab der Reichstagswahl vom 29. März 1936 diesen das Wahlrecht, was sich in der Verwaltungspraxis der Wahlämter als nicht immer einfach erwies. ${ }^{32}$ Dagegen war bei der Volksabstimmung vom 10. April 1938 dieser Ausschluss schon zur Routine geworden: „Juden - auch Geltungs-Juden, sind zur Wahl nicht zuzulassen. Die etwaige Eintragung ist mit Vermerk zu versehen. Falls sie glauben, stimmberechtigt zu sein, sind sie an das Wahlamt zu verweisen“, so die Zusammenfassung einer Besprechung in Kiel, in der es außerdem hieß: „Die Frage der rassischen $\mathrm{Zu}$ gehörigkeit der Zigeuner ist immer noch nicht eindeutig geklärt. Die Zigeuner sind gegebenenfalls auf die rassischen Voraussetzungen hinzuweisen. Es ist aber zu bedenken, daß auch unter den Zigeunern Alte Kämpfer sind." 33 Im selben Jahr diente der ohne juristische Grundlage durch den Reichsführer SS, Heinrich Himmler, bestimmte Ausschluss der politischen Schutzhäftlinge ${ }^{34}$ dazu, den KZ-Häftlingen kein Ventil für ihren Dissens zu erlauben, denn damit hatte man im November 1933 noch einschlägige negative Erfahrungen sammeln müssen. ${ }^{35}$ Die 24 bzw. 26 Gegenstimmen der 70 Häftlinge bewertete der Verantwortliche im KZ Glückstadt so: „Das Ergebnis zeigt, daß rund ein Drittel aller Schutzhäftlinge immer noch nicht begriffen hat oder begreifen will, um was es heute geht. Leider sind die Namen der Unbelehrbaren ja nicht feststellbar."36 Im April 1938 hatten die Machthaber natürlich kein Interesse mehr daran, das Wahlrecht der Schutzhäftlinge in die Praxis umzusetzen, was Wahlkabinen in den großen Konzentrationslagern und ein möglicherweise bis ins Ausland reichendes Echo auf die dort zu erwartende relative Abstimmungsniederlage der NS-Diktatur bedeutet hätte.

In der Frage von Wahlrecht und Wahlpflicht gab es formal 1933 bis 1938 zwar keine Änderung der Gesetzeslage, doch konnten die Nationalsozialisten an die schon unter den Parteien der Weimarer Republik vertretene Vorstellung anknüpfen, dass

31 Bundesarchiv Berlin R 1501/5350, B1. 63. - Vgl. P. Hubert, Uniformierter Reichstag. Die Geschichte der Pseudo-Volksvertretung 1933-1945, 1992, S. 241, 248 f.

32 RGB1, Teil 1, 1936. S. 133, 7.3.36, „Gesetz über das Reichstagswahlrecht“. - Zu den Problemen in den Kommunen siehe Omland, Du wählst (Fn. 10), S. 130 ff.

33 Stadtarchiv Kiel 40679, Besprechung der Wahlvorstände und des Oberbürgermeisters vom 8. April 1938.

34 Die Gestapo wies in einem Schreiben am 31.3.38 auf das Wahlrecht von Schutzhäftlingen hin und revidierte dies am 8.4.38. Vgl. dazu Stadtarchiv Kiel 40679, Schreiben des Regierungspräsidiums Schleswig an alle Oberbürgermeister vom 4.4.38 sowie Landesarchiv SchleswigHolstein [= LAS] 309/22674.

35 Vgl. dazu das Dokument aus dem KZ Glückstadt bei Omland, Du wählst (Fn. 10), S. 58.

36 LAS 309/22574. Quelle abgedruckt in Omland, Du wählst (Fn. 10), S. 58. 
Wahlrecht genuin Wahlpflicht sei, ${ }^{37}$ und damit die zwangsweise Mobilisierung der Wahlberechtigten während ihrer Herrschaft legitimieren. Die Weiterentwicklung der Wahlschleppdienste der Weimarer Republik zu einem Instrument des Aufsuchens und der Nötigung der Nichtwähler, zur Wahl zu gehen, war in dieser Logik nur konsequent.

Die Kehrseite der Medaille einer solchen möglichst breiten Mobilisierung der Wahlberechtigten durch eine faktische Wahlpflicht war allerdings die Unglaubwürdigkeit der (zu hohen) Ergebnisse: Wenn alle zur Abstimmung gingen und sich gleichzeitig dort keine Opposition zeigte bzw. der zu erwartende Dissens eines Teils der Bevölkerung sich dort nicht widerspiegelte - so gerade 1936 und 1938 - dann war dies weder den eigenen Anhängern noch der Bevölkerung vermittelbar. ${ }^{38}$

\section{Instrumentalisierung der Abstimmungen durch die NS-Politik}

Alle Abstimmungen im NS-Staat wurden kurzfristig und aus rein instrumentalistischen Gründen durchgeführt. Ziel war es, selbstverschuldete außen- bzw. innenpolitische Krisen mittels Akklamation im Sinne Deutschlands zu beeinflussen. Die propagierte große Zustimmung bei den Urnengängen diente gegenüber den westlichen Demokratien zur Demonstration der (vermeintlichen) Einheit von Führung und Volk im Sinne der Volksgemeinschaftsideologie und nach Innen der Legitimation der Herrschaft gegenüber der Bevölkerung.

Die politische Instrumentalisierung aller Urnengänge ist sowohl an den Abstimmungsterminen als auch an den Inhalten der Plebiszite abzulesen: ${ }^{39}$ Es begann im Herbst 1933, als das Deutsche Reich am 14. Oktober die Genfer Abrüstungskonferenz verließ, da es die geheime deutsche Aufrüstungspolitik durch britische Vorschläge zur Abrüstungskontrolle gefährdet sah. Um hiervon unabhängig handeln zu können, trat Deutschland aus dem Völkerbund aus ${ }^{40}$ und wollte diesen Schritt durch eine Volksabstimmung und gleichzeitige Reichstagswahlen ${ }^{41}$ absichern. Ähnlich

37 In den Wahlkämpfen der Weimarer Republik propagierten dies sowohl die Parteien als auch die Regierung. Vgl. Itzehoer Nachrichten 4.11.1932, „Nichts halte Dich! Gehe wählen!“ Husumer Nachrichten 30.7.1932, „Halt! Erst wählen!“ - Marner Zeitung 19.5.1928, „Wahlrecht ist Wahlpflicht".

38 Vgl. Deutschlandberichte der Sozialdemokratischen Partei Deutschlands 1934-1940, 1936, S. 319, 449 und 1938, S. 426 ff.

39 Vgl. für Schleswig-Holstein Omland, Du wählst (Fn. 10), S. 39 ff. (1933), 89 (1934), 129 (1936), 161 und S. 187 (1938).

40 Altonaer Nachrichten 14.10.1933: „Deutschland tritt aus dem Völkerbund aus. - Vgl. L. Herbst, Das nationalsozialistische Deutschland 1933-1945, 1996, S. 104-110. Am 14.10.1933 erfolgte die Verkündung des Austritts, am 21.10.1933 die schriftliche Begründung, und erst zwei Jahre später war der Austritt vollzogen.

41 RGB1. 1933 I, S. 729: „,Verordnung des Reichspräsidenten über die Auflösung des Reichstags. Verordnung über die Neuwahl des Reichstags“. - Kieler Zeitung 15.10.1933: „Die Auflösung des Reichstags“. - Akten der Reichskanzlei, Regierung Hitler, Bd. 2: 12. September 1933 bis 27. August 1934, 1983, S. 906. 
sah es im März 1936 aus, als mit einer Reichstagswahl die außenpolitische Krise nach dem Einmarsch ins entmilitarisierte Rheinland und dem damit einhergehenden Bruch des Versailler Vertrages gemildert, ${ }^{42}$ und 1938, als die Annexion Österreichs - auch und gerade durch die dortige Abstimmung! - legitimiert werden sollte. ${ }^{43}$ Die „Sudetendeutsche Ergänzungswahl“ am 4. Dezember 1938 hatte zwar ein ähnliches Ziel, ${ }^{44}$ trat aber angesichts der wenigen sudetendeutschen Bürger im Reichsgebiet jenseits des besetzten Teils der Tschechoslowakei deutlich hinter anderen innenpolitischen Ereignissen im „Altreich“ zurück. ${ }^{45}$ Lediglich am 19. August 1934 wurde eine innenpolitische Frage zur Abstimmung gestellt: die Übernahme des Reichspräsidentenamtes durch Hitler nach dem Tod des Amtsinhabers Paul von Hindenburg. ${ }^{46}$ Diese Volksabstimmung fand sowohl vor dem Hintergrund der Ermordung der SA-Führung um Ernst Röhm (30. Juni-1./2. Juli) ${ }^{47}$ als auch der sich seit Ende

42 RGB1. 1936 I, S. 133: „Verordnung des Führers und Reichskanzlers über die Auflösung und Neuwahl des Reichstags“; S. 134: „Erste Verordnung zur Reichstagswahl“.

43 Nordische Rundschau 12./13.3.1938: „Schuschniggs Verrat: Der Führer rettet Deutsch-Österreich vor einem neuen Bürgerkrieg“, ebd. „Ganz Kiel feiert heute abend Österreichs nationale Erhebung“. - RGB1. 1938 I, S. 237: 13.3.1938: „Gesetz über die Wiedervereinigung Österreichs mit dem Deutschen Reich.“ - S. 249, 16.3.1938: „Verordnung zum Gesetz über die Wiedervereinigung Österreichs mit dem Deutschen Reich.“- Sowohl die österreichische als auch die deutsche Regierung hatten per Gesetz den „Anschluss“ legitimiert, womit die Abstimmung nur noch als eine nachträgliche Legitimierung des Faktischen gedacht gewesen war. Vgl. Jung, Plebiszit (Fn. 28), S. $109 \mathrm{ff}$.

44 Laut Hubert, Reichstag (Fn. 31), S. 149 ff. stimmte Hitler der Wahl aus rein taktischen Gründen zu. - RGBl. 1938 I, S. 1636 (21.11.38, Gesetz über die Wiedervereinigung der sudetendeutschen Gebiete mit dem Reich), RGB1. 1938 I, S. 1567.

45 Parallel fanden im Deutschen Reich die Sammlungen für das Winterhilfswerk vor dem Weihnachtsfest statt, so dass die Reichstagswahl auf den Titelseiten eine Nebenrolle spielte. Vgl. Kieler Neueste Nachrichten 5.12.1938: „Großartiger Sieg des deutschen sozialen Gedankens: Die phantastische Summe von über 15 Millionen Mark erreicht“ und „Überwältigendes sudetendeutsches Bekenntnis zu Großdeutschland“.

46 RGB1. 1934 I, S. 747, 1.8.1934, Gesetz über das Staatsoberhaupt des Deutschen Reichs. - Ebd., S. 751, 2.8.1934, Erlaß des Reichskanzlers zum Vollzug des Gesetzes über das Staatsoberhaupt des deutschen Reichs vom 1. August 1934. - Ebd., S. 745. 2.8.1934, Nachricht vom Ableben des Reichspräsidenten Generalfeldmarschall von Hindenburg. - Ebd., S. 752, 2.8.1934, Beschluß der Reichsregierung zur Herbeiführung einer Volksabstimmung. Kundgebung der Reichsregierung an das deutsche Volk. - Ebd., S. 757, 3.8.1934, VO zur Durchführung der Volksabstimmung über das Staatsoberhaupt des Deutschen Reichs. Erste VO zur Volksabstimmung über das Staatsoberhaupt des deutschen Volkes - Vgl. Akten der Reichskanzlei (Fn. 41), S. 1382 ff., Dokumente 381, 382 und 383. - Vgl. Jung, Plebiszit (Fn. 28), S. 61 ff. (Formal war es keine Volksabstimmung nach dem entsprechenden Gesetz mehr). - Zu Schleswig-Holstein vgl. Omland, Du wählst (Fn. 10), S. 89 f.

47 Vgl. Herbst, Deutschland (Fn. 40), S. 116. - Für Schleswig-Holstein vgl. die Anweisungen in LAS 301/4565 und die Berichterstattung in der Schleswig-Holsteinischen Tageszeitung vom 2.7.1934: „Es lebe der Führer!“, „,Der Schlag gegen Meuterer und Reaktion“. Ebd. 3.7.1934: „Die Idee hat gesiegt! Gegen unsinnige Gerüchte!“ 
Mai/Anfang Juni artikulierenden kirchlichen Opposition gegen das NS-Regime statt. $^{48}$

Bei allen Abstimmungen handelte das Regime immer sehr kurzfristig, ohne längere Planungen und hielt sich nicht an gesetzlich vorgesehene Fristen, etwa was die Dauer der Legislaturperioden des Reichstags anging. ${ }^{49} \mathrm{Da}$ es freie öffentliche Meinungsäußerungen nicht zulassen konnte, sollte durch die Abstimmungen gegenüber dem Ausland eine positive Stimmungslage des „Volkes“ gegenüber dem Regime suggeriert werden. Während das überraschend hohe Ergebnis im November 1933 dies noch bewirkte und auch die relative Niederlage 1934 glaubwürdig war, verkehrten die Abstimmungen von 1936 und 1938 dieses Anliegen in sein Gegenteil. Die verkündeten Endergebnisse mit reichsweit über 97 Prozent Zustimmung aller Wahlberechtigten waren weder nach außen noch nach innen - auch innerhalb der NS-Anhängerschaft - glaubwürdig zu vertreten. ${ }^{50}$

Die Instrumentalisierung von Plebisziten lässt sich auch daran ablesen, dass Hitlers Ankündigung, jährlich Volksabstimmungen durchzuführen, nach der relativen Niederlage 1934 stillschweigend nicht mehr umgesetzt wurde. ${ }^{51}$ Dementsprechend führte im Februar 1935 eine Anfrage der Stadt Kiel beim Regierungspräsidium zu Irritationen: „Da alljährlich eine Volksabstimmung durchgeführt werden soll, bitte ich um Nachricht, ob demnächst mit dem Eingang der Stimmzettelumschläge gerechnet werden kann. “"52

\section{Wahlkampf und Wahlpropaganda einer ,,Volksgemeinschaftspartei “}

Die Wahlpropaganda lag in der Verantwortung der NSDAP, die aus damaliger Sicht einen hochmodernen Wahlkampf unter Einsatz aller verfügbaren Massenmedien führte. Im Sinne einer „Volksgemeinschaftspartei“ versuchte die Partei, alle Wahlberechtigten freiwillig, durch sozialen Druck und mit Zwang zu einem „Ja“ bei den Abstimmungen zu bewegen.

Ein Vergleich aller Plebiszite und Wahlen von 1933 bis 1938 zeigt, dass ihnen allen der relativ kurze Zeitraum gemeinsam war, in dem die NSDAP Propaganda

48 Die „Erste Reichs-Bekenntnissynode in Barmen“ der Protestantischen Kirchen fand am 29.-31. Mai 1934 statt und wandte sich gegen den Totalitätsanspruch des NS-Regimes. Die katholischen Bischöfe verabschiedeten am 7. Juni einen Hirtenbrief gegen die „Irrtümer der Zeit", der vor der Verteilung beschlagnahmt wurde. Vgl. C. Studt, Das Dritte Reich in Daten, 2002, S. 38.

49 So etwa hätten nach den Reichstagswahlen vom 5. März 1933 Neuwahlen im Jahr 1937 und nach den Reichstagswahlen vom 29. März 1936 Neuwahlen im Jahr 1940 stattfinden müssen.

50 Vgl. die Quellenbelege bei Omland, Du wählst (Fn. 10), S. 152 (1936), 185 (1938).

51 Jung, Plebiszit (Fn. 28), S.58 und Deutschland-Berichte (Fn. 38), 1938, S. 394. Noch 1934 hatte Reichsinnenminister Frick darauf im Wahlkampf abgehoben, vgl. dazu Altonaer Nachrichten 13.8.1934, „Sinn der Volksabstimmung“.

52 LAS 309/22573. Schreiben des Oberbürgermeisters von Kiel an das Regierungspräsidium, 14.2.1935. 
betreiben konnte. Maximal waren es vier Wochen, ${ }^{53}$ in denen der öffentliche Raum vollständig von den Nationalsozialisten ausgefüllt werden konnte. Der kurze „Wahlkampf"-Zeitraum sollte im Oktober/November 1933 nicht zum Problem werden, doch beging das NS-Regime im August 1934 nach dem Tod des Reichspräsidenten Hindenburg einen entscheidenden Fehler. Es setzte den Abstimmungstermin über die Zusammenlegung des Reichspräsidentenamtes mit dem Reichskanzleramt Hitlers zu kurzfristig an. Nach einer Woche Staatstrauer verblieb nur etwas mehr als eine weitere Woche für die Abstimmungskampagne und man verzichtete fast vollständig auf die Mobilisierung mit Aufmärschen, Versammlungen und öffentlicher Propaganda. So waren selbst Sprechabende von Gau- und Kreisrednern verboten und auch Gauleiter Lohse hielt lediglich eine kurze Radioansprache. ${ }^{54}$ Ziel war es, sich als ,pietätvoll“ im Angesicht von Hindenburgs Tod darstellen zu können. Deshalb setzte man sehr einseitig auf die Zeitungspropaganda und einige wenige öffentliche Auftritte oberster Partei- und SA-Führer sowie von Regierungsmitgliedern. In Kiel trat Rudolf $\mathrm{He} ß$ auf, der Hitler als einen „Messias“ der Deutschen feierte und an die SA gewandt unmissverständlich drohte: „Der 30. Juni zeigte den Geführten, daß sie die menschliche Güte des Führers, seine vorsichtige und weise Führung nicht mißverstehen dürfen, daß sie daraus nicht das Recht ableiten dürfen, sich über den Willen des Führers hinwegzusetzen oder gar Verrat an ihm und seiner Sache zu üben. Der 30. Juni beugt vor." ${ }^{55}$ Doch die gerade einmal einen Monat zurückliegende Ermordung der SA-Führung ${ }^{56}$ und die nicht öffentlich äußerbare Kritik an der Zusammenlegung der beiden Staatsämter führten zu einer selbstverschuldeten, vermeidbaren relativen Niederlage Hitlers. Die Zurückhaltung bei der Propaganda erwies sich als ein solcher Fehlschlag, dass ab 1936 der Wahlkampf vollständig in die Hände von Reichspropagandaminister Joseph Goebbels gelegt wurde und die beiden nachfolgenden Wahlkämpfe 1936 und 1938 so hochprofessionell organisiert wurden, dass sich solche Fehler nicht wiederholten.

In der NS-Diktatur griff die Partei für den „Wahlkampf“ und die Propaganda auf ihre Erfahrungen aus der Weimarer Republik zurück: Sprechabende der NSDAP, Massenversammlungen, Demonstrationszüge durch die Straßen - auch und gerade auf dem Land -, Bildpropaganda durch Karikaturen, Fotomontagen und Plakate, die Nutzung von Symbolen wie Uniformen und Fahnen sowie Wahlwerbung in der Presse, im Kino und dem Rundfunk. ${ }^{57}$ Neu waren die Möglichkeit, auch in den Be-

53 Zeiträume für die Propaganda: 14.10 .1933 bis $12.11 .1933 ; 2.8 .1934$ bis 19.8 .1934 (davon eine Woche Staatstrauer); 7.3.1936 bis 29.3.1936; 18.3 .1938 bis 10.4 .1938 .

54 Omland, Du wählst (Fn. 10), S. 94 - Nordische Rundschau 16.8.1934, „Gauleiter Lohse zum 19. August".

55 Kieler Neueste Nachrichten 16.8.1934: „Rudolf Heß wirbt für den Führer“.

56 Vgl. Wendt, Deutschland (Fn. 21), S. 123 ff. - Im Wahlkampf wandte sich Rudolf Heß direkt auch an SA-Männer, um sie für ein Ja zu gewinnen. Vgl. Omland, Du wählst (Fn. 10), S. 95 f. über einen Auftritt in Kiel am 14.8.1934.

57 Ein Vergleich mit den bei Paul, Aufstand (Fn. 15), für die Weimarer Republik aufgeführten Mechanismen und Mitteln der NS-Propaganda belegt, dass die Partei ihre bekannten Möglichkeiten um die aus der Macht im Einparteienstaat gewonnenen ergänzte. Vgl. auch P. Rei- 
trieben und den Schulen für die Staatspartei und deren Führer zu werben sowie die Presselenkung. Zentrales Instrument für letztere waren die Treffen des Reichspropagandaministeriums in Berlin mit den führenden deutschen Zeitungen, in denen diese vorgeschrieben bekamen, was, wo und wie kommentiert in der Presse abgedruckt werden sollte. Während es noch 1933 und 1934 zu einer Reihe von Abweichungen von diesen Sprachregelungen kam, war dies 1936 und 1938 praktisch nicht mehr der Fall, und die Presseanweisungen hatten sich durchgesetzt. ${ }^{58}$

Die Zeitungen brachten wiederkehrende Parolen und Artikel, berichteten über den regionalen und überregionalen Wahlkampf und überschlugen sich in Ergebenheitsadressen an den „Führer“. Das öffentliche Bild wurde durch Spruchbänder, wahlpropagandistische Installationen und (überdimensionale) Hitler-Portraits bestimmt. Der Wahlkampf schloss mit großen Aufmärschen zu zentralen Plätzen, der Übertragung einer Rede Hitlers, einem Dankgebet und dem abschließenden Schwur auf „Führer, Volk und Vaterland“ ab. ${ }^{59}$

\section{7. „Deine Stimme dem Führer"60}

Durch eine Personifizierung des Wahlkampfes auf Adolf Hitler funktionierte das Regime jeden Urnengang zu einer Akklamation für seinen „Führer“ um. Hitler wurde in der Wahlpropaganda zum „Messias“ der Deutschen stilisiert, an dessen Politik sich pseudoreligiöse Heilserwartungen knüpften. ${ }^{61}$

Die NSDAP und die Reichsregierung setzten bei allen Urnengängen auf die Zustimmung der Bevölkerung zur Person Adolf Hitlers, so dass jede Abstimmungsfrage zu einer abzutragenden „Dankesschuld“ für dessen Einsatz für Deutschland funktionalisiert werden konnte. Die Wahlberechtigten sollten so nicht mehr für oder gegen eine bestimmte Sachfrage bzw. die NSDAP stimmen können, sondern nur noch für oder gegen den „Führer“ persönlich, womit eine zusätzliche psychologische Hemmschwelle für Gegenstimmen aufgebaut wurde. Der Rückgriff erfolgte direkt bei Parolen für den Wahlkampf wie etwa „Volksgenosse! Der Führer braucht auch Deine Stimme! Versage sie ihm nicht: Stimme mit Ja!“62 (1933) oder „An Dich

chel, Der schöne Schein des Dritten Reiches. Gewalt und Faszination des deutschen Faschismus, 2. Aufl. 2006, S. $139 \mathrm{ff}$. und $198 \mathrm{ff}$.

58 Vgl. H. Bohrmann/G. Toepser-Ziegert u.a. (Hrsg.), Nationalsozialistische Presseanweisungen der Vorkriegszeit. Edition und Dokumentation. Institut für Zeitungsforschung Stadt Dortmund. 1984, 1985, 1993, 1998 und 1999. (Edierte Jahrgänge: 1933-1938).

59 Vgl. dazu Omland, Du wählst (Fn. 10), S. 42 ff. (1933), 93 ff. (1934), 132 ff. (1936), 166 ff. (1938).

60 Altonaer Nachrichten 27.3.1936.

61 Vgl. für Schleswig-Holstein: Omland, Du wählst (Fn. 10), S. 46 ff. zum Besuch Adolf Hitlers im Wahlkampf am 6. November 1933 in Kiel. - Grundsätzlich: I. Kershaw, Führer und Hitlerkult, in: W. Benz/H. Graml/H. Weiß (Hrsg.), Enzyklopädie des Nationalsozialismus, 1997, S. $22 \mathrm{ff}$.

62 Norddeutsche Nachrichten 11.11.1933. 
glauben wir - Adolf Hitler“ (1934) ${ }^{63}$. Hinzu kamen Fotomontagen, ${ }^{64}$ Berichte ${ }^{65}$ und Appelle in Wahlkampfreden: „Die Welt soll hören, was wir denken: die Welt soll vernehmen, was wir glauben: Ein Volk, ein Reich, ein Führer.“66

Eine Anmutung dessen, was dieser Führerkult beinhaltete, erhält man beim Hören der Originalaufnahme einer knapp anderthalbstündigen Rede Hitlers in der Kieler Nordostseehalle im November 1933. Unter den Klängen des Badenweiler Marsches ging dieser durch die Reihen im Saal, es gab auf- und abebbende Wellen von „Heil!““Rufen und eine minutenlange Stille, in der die Menge darauf wartete, dass er mit seiner Ansprache begann. ${ }^{67}$ Die Tatsache, dass die Menge während der Rede auch dann in Jubelrufe ausbrach, wo dies rhetorisch nicht ganz passte, zeigt auf, dass sich die 20.000 Anwesenden nicht lediglich passiv inszenieren, sondern sich auch von ihrer Begeisterung tragen ließen. ${ }^{68}$

\section{Inszenierung des Abstimmungstages}

Die NSDAP zelebrierte den Sonntag der Abstimmung bewusst als einen Festtag für die Volksgemeinschaft. Ziel war es, das Gefühl der Wahlberechtigten zugunsten des Regimes zu beeinflussen und die vorhandene Opposition unter ihnen durch eine Mischung von „schönem Schein“ und sozialer Kontrolle zugunsten des neuen Staates zu beeinflussen. ${ }^{69}$

Am Sonntagmorgen zogen Spielmannszüge der SA oder auch der Hitler-Jugend durch die Straßen und weckten die Bevölkerung auf. Platzkonzerte sollten den Festtagscharakter unterstreichen, geschmückte Straßenzüge, öffentliche Installationen von Innungen oder auch der Partei sollten die positive Stimmung unter den Wahlberechtigten befördern. Die Zeitungen forderten zum frühen Besuch der Abstimmungslokale auf, lange Warteschlangen vor diesen unterstrichen das Gemeinschaftsgefühl und die behauptete Einheit in der Volksgemeinschaft. Das gemeinsame Aufmarschieren von Gruppen zum Wahllokal - etwa 1938 der wahlberechtigten Österreicher in Kiel - verstärkte den Wahlzwang und produzierte gleichzeitig Bilder einer Geschlossenheit der Volksgemeinschaft. Nach dem Urnengang erhielten die

63 Nordische Rundschau 15.8.1934.

64 Nordische Rundschau 18./19.8.1934, „Im Volke geboren, zum Führer erkoren. Alle sagen: Ja!“

65 Nordische Rundschau 18./19.8.1934, „Im Banne des Führers“ [Abschlussrede Hitlers in Hamburg 1934].

66 Rede von SA-Obergruppenführer Meyer-Quade, Kieler Neueste Nachrichten 7.4.1938: „Die ganze Welt soll unser Bekenntnis hören!“

67 Deutsches Rundfunkarchiv, Archiv-Nr. C 7423. - Vgl. W. Roller (Bearb.), Tondokumente zur Kultur- und Zeitgeschichte 1933-1935. Ein Verzeichnis, 2000, S. 165: 382. Kundgebung in der Kieler Nordostseehalle anlässlich der Volksabstimmung am 12. November 1933. - Der Schlussteil der Rede ist abgedruckt bei Omland, Du wählst (Fn. 10), S. 50.

68 Vgl. Omland, Du wählst (Fn. 10), S. 48.

69 Vgl. zum Folgenden Omland, Du wählst (Fn. 10), S. 52 ff. (1933), 101 ff. (1934), 141 ff. (1936) und 173 ff. (1938). - Vgl. grundsätzlich Reichel, Schein (Fn. 57), S. 262 ff. 
Wähler eine Anstecknadel, deren Tragen die soziale Kontrolle der Nichtwähler, die diese Wahlplakette noch nicht besitzen konnten, erhöhte. Üblicherweise traf man sich abends zur Verkündung der Zwischenergebnisse vor den Zeitungsverlagen oder hörte in Gaststätten und extra dafür eingerichteten Versammlungsräumen deren Übertragung im Radio, was das Gemeinschaftsgefühl ebenso befördert haben dürfte.

\section{Wählertäuschung, Wahlgeheimnisbruch, Wahlfälschung}

Die Reichsregierung und die Verwaltung versuchten den Schein von „rechtstaatlichen" Abstimmungen möglichst lange aufrecht zu erhalten, doch bestimmten zunehmend die NSDAP bzw. ihre Gliederungen und Verbände die Wirklichkeit bei den Plebisziten und Reichstagswahlen. Zudem manipulierte das NS-Regime jederzeit das Stimmergebnis zu seinen Gunsten: im November 1933 durch eine Veränderung in der Auszählung der ungültigen Stimmen, 1936 durch eine staatlich angeordnete - und öffentlich gewordene - Wahlfälschung sowie 1936 den Ausschluss von „Juden“ und 1938 den Ausschluss von politischen Schutzhäftlingen. ${ }^{70}$

Die Partei ließ durch den „Wahlschleppdienst“ der SA die Wahlberechtigten zu Hause aufsuchen und nötigte diese, an die Urne zu gehen. Sie besetzte bis spätestens 1936 sämtliche Positionen in den Wahllokalen, und die Stimmauszählung fand im Laufe der Zeit unter völligem Ausschluss der Öffentlichkeit statt. Die NSDAP konnte so nicht nur Wahlfälschung betreiben, sondern auch anhand der Wählerverzeichnisse und Stimmscheinlisten die möglichen Wahlboykotteure identifizieren und verfolgen lassen. Die Angst vor der Denunziation als nicht wählender „Landesverräter“ und Gerüchte über die Re-Identifizierung der Nichtwähler anhand der Wählerverzeichnisse waren Gründe für die sehr hohe Wahlbeteiligung im Nationalsozialismus. Insgesamt nahm die soziale Kontrolle vor und im Wahllokal von Abstimmung zu Abstimmung zu und erhöhte den Druck, mit „Ja“ zu stimmen, so sehr, dass von einer ,freien“ Wahlentscheidung spätestens ab 1936 nicht mehr gesprochen werden kann.

Die erste Maßnahme zur Veränderung des Stimmergebnisses war im November 1933 der Versuch der Wählertäuschung vor dem Hintergrund einer Doppelabstimmung. Die Wahlberechtigten erhielten je einen Stimmzettel für die Reichstagswahl und für die Volksabstimmung, aber nur einen Wahlumschlag. Während bei vorherigen Doppelwahlen die Nichtabgabe eines Stimmzettels als ungültige Stimme gewertet worden war, galt dieses Verhalten nun als Stimmenthaltung. Formal korrekt hatte die Regierung diese Änderung durch die Presse bekannt gegeben, doch ob solche Notizen wirklich von den Wählerinnen und Wähler bemerkt wurden, muss offen bleiben. ${ }^{71}$ Da der Stimmzettel zur Reichstagswahl nur die Möglichkeit bot, die

70 Zum Folgenden Omland, Du wählst (Fn. 10), S. 52 ff. (1933), 101 ff. (1934), $130 \mathrm{ff}$. und $141 \mathrm{ff}$. (1936), $161 \mathrm{ff}$. und $174 \mathrm{ff}$. (1938).

71 Vgl. Nordische Rundschau 27.10.1933: „Die Abstimmungszeiten...“. - Nordfriesische Rundschau 27.10.1933: „Einheitliche Richtlinien für die Feststellung des Wahlergebnisses“. Schleswig-Holsteinische Tageszeitung 26.10.33: „Wie wird gewählt?“. - Stadtarchiv Bad Ol- 
NSDAP anzukreuzen, mussten Gegenstimmen durch Beschriftung, Nichtausfüllen oder eben durch Weglassen des Stimmzettels im Wahlumschlag bekundet werden. Letzteres galt aber nicht mehr als ungültige Stimme, sondern lediglich als Enthaltung. Diese Wahlenthaltungen flossen nicht in das offizielle Reichstagswahlergebnis ein, das wie üblich in Prozent der abgegebenen (gültigen) Stimmen ausgewiesen wurde. Die Höhe der so bei der Reichstagswahl ,weggefallenen“ Gegenstimmen zur NSDAP schwankte von Wahllokal zu Wahllokal und dürfte für Schleswig-Holstein insgesamt bei knapp 15.000 Stimmen gelegen haben, was knapp 1,3 Prozent der Wahlberechtigten entsprach. ${ }^{72}$

Der Bruch des Wahlgeheimnisses ist ebenfalls belegbar: So wollte etwa 1934 in Luhnstedt/Kreis Rendsburg der Wahlvorstand durch Knicken von Stimmzetteln mögliche Gegner Hitlers bei der Stimmauszählung identifizieren. ${ }^{73}$ Da es umgekehrt gleichzeitig Quellenbelege gibt, die die Wahrung des Wahlgeheimnisses aufzeigen, ${ }^{74}$ ist eine Generalisierung auf alle Wahllokale nicht möglich, denn entscheidend war die konkrete Situation vor Ort.

Nicht unerwähnt soll die staatlich angeordnete Wahlfälschung bei der Reichstagswahl 1936 bleiben, ${ }^{75}$ der in Schleswig-Holstein zwei von drei oder sogar drei von vier Gegenstimmen zum Opfer fielen. Dadurch, dass sie öffentlich bekannt wurde, delegitimierte sie das Endergebnis, wie Reaktionen in den Betrieben und unter den NS-Anhängern aufzeigen. Seitens der schleswig-holsteinischen Bevölkerung waren aber auch schon 1933 und 1934 Zweifel an der Rechtmäßigkeit der Wahlergebnisse geäußert und Mutmaßungen über Wahlfälschungen angestellt worden. ${ }^{76}$

\section{Exkurs: Zur Vergleichbarkeit von Wahlergebnissen}

In der Regel werden zum Vergleich von Wahlergebnissen die abgegebenen gültigen Stimmen herangezogen, denn diese bestimmen die Anzahl der Mandate in den Parlamenten. Dagegen greift die historische Wahlforschung für die vergleichende Analyse von Ergebnissen auf einen den gesamten Wahlkörper einschließenden Ansatz

desloe, Akte 858, Blatt 45, Vermerk des Magistrats, 26.10.1933. - Stadtarchiv Neumünster Akte 3194, Magistrat an die Wahlvorsteher, 26.10.1933.

72 In Kiel hat es in einem Wahllokal Stimmrückgänge in Höhe von knapp 19\% gegeben: Omland, Du wählst (Fn. 10), S. 53 f. und 72 f. - Die größte Differenz ist bisher für ein Wahllokal in Lübeck mit 28,5\% weniger abgegebenen Stimmzetteln für die Reichstagswahl als für die Volksabstimmung nachweisbar. Zu Details ders., ,Jeder Deutsche stimmt mit Ja!“ Die erste Reichstagswahl und Volksabstimmung im Nationalsozialismus am 12. November 1933, in: Zeitschrift der Gesellschaft für Schleswig-Holsteinische Geschichte 131 (2006), S. 133 (162-168).

73 Vgl. den Briefwechsel zwischen dem Rechtsanwalt und dem Regierungspräsidium in LAS 309/22738. - Siehe auch Omland, Du wählst (Fn. 10), S. 103; 1936 ist ebenfalls ein Wahlgeheimnisbruch belegt: ebd., S. 145 und Abbildung S. 145.

74 Omland, Du wählst (Fn. 10), S. 145.

75 Zum Folgenden siehe Omland, Du wählst (Fn. 10), S. 144 ff.

76 Vgl. Omland, Du wählst (Fn. 10), S. 60 und 108. 
zurück und bezieht auch die Nichtwähler mit ein. Die Prozentuierung auf die Zahl der Wahlberechtigten ermöglicht es - unabhängig von Schwankungen der Wahlbeteiligung - Ergebnisse miteinander zu vergleichen. ${ }^{77}$

Ein kurzes Beispiel soll das ansonsten auftretende Problem verdeutlichen: In einer Gemeinde gibt es 100 Wahlberechtigte, von diesen beteiligen sich 10 an der Wahl und diese stimmen alle für Partei A (= 100 Prozent der abgegebenen Stimmen). Bei der nächsten Wahl geben 20 Wahlberechtigte ihre Stimme ab, davon 10 für Partei A und 10 für Partei B (jeweils 50 Prozent der abgegebenen Stimmen). Ein Vergleich beider Ergebnisse in abgegebenen gültigen Stimmen würde einen Rückgang um 50 Prozent für Partei A ergeben, obwohl diese Partei tatsächlich ihr Ergebnis hat halten können: bei jeder Wahl gaben jeweils 10 Wahlberechtigte ihre Stimme dieser Partei. Außerdem wird in diesem zugespitzten Beispiel die starke Bedeutung der Wahlenthaltung und der Nichtwählermobilisierung deutlich: Partei B konnte durch die Mobilisierung der ehemaligen Nichtwähler einen Wahlerfolg verbuchen.

\section{Schätzungen zu den Wählerwanderungen: das Logit-Modell}

Die im Folgenden genannten Schätzungen zu den Wählerwanderungen basieren auf dem Logit-Modell von Sören R. Thomsen.$^{78}$ Dieser hat ein multivariables wahlstatistisches Programm entwickelt, das - sehr verkürzt ausgedrückt - die Übergangswahrscheinlichkeiten der Wählerschaften zwischen den Parteien unter Verwendung der Referenzkategorie der Nichtwähler berechnet. ${ }^{79}$ Grundsätzlich sind Schätzungen nach dem Logit-Modell den zu Fehlinterpretationen führenden Analysen aufgrund der Berechnung von Korrelationskoeffizienten ${ }^{80}$ oder auch dem Swing-Index (Sigurd Matz) als Modell für die Wechselwählerbereitschaft der Parteien vorzuziehen. ${ }^{81}$ Das Logit-Modell hat den Vorteil, dass es anhand von Wahlen überprüft werden kann, bei denen aktuelle Umfragen zu Wählerwanderungen vorliegen. ${ }^{82}$

77 Vgl. dazu Falter, Hitlers Wähler (Fn. 14), S. 63 f.

78 S.R. Thomsen, Danish Elections 1920-1979. A Logit Approach to Ecological Analysis and Inference, 1987.

79 Wählerwanderungen können nur berechnet werden, wenn nicht nur die abgegebenen Stimmen, sondern auch die Anzahl der Wahlberechtigten überliefert ist. So etwa für die Wahllokale des Stadtkreises Neumünster (39.895 Einw./Juni 1933): F. Omland, ,Jeder Deutsche sagt morgen: Ja!“. Neumünster und die erste Reichstagswahl und Volksabstimmung im Nationalsozialismus am 12. November 1933, 2007, S. 35-37.

80 Vgl. zu diesen Problemen Falter, Hitlers Wähler (Fn. 14), S. 55 ff.

81 S. Matz, Zeitgemäßes Campaigning in der Bundesrepublik Deutschland: Empirische und evaluative Hinweise zum Swing-Index, einem neuen Instrument des ,micro targeting“, in: Duisburger Beiträge zur Soziologischen Forschung 1 (1991), S. 1-18. - Amt für Bürgerservice und Informationsverarbeitung (Hrsg.), Stammwählerschaft und Wechselwähler in den Freiburger Wahlbezirken. Der Swing-Index als Instrument zur Messung der Wahlstabilität. = Statistischer Infodienst 22. März 2006, S. 3-9.

82 Amt für Statistik und Einwohnerwesen. Statistischer Infodienst. Schätzung der Freiburger Wählerwanderung zwischen den Landtagswahlen 1996/2001. Eine ökologische Analyse auf der Grundlage eines Logit-Modells. 15. Juni 2001. 
10. Die Wahlergebnisse als Gradmesser für die Integration in die Volksgemeinschaft und als Gradmesser der Ablehnung des NS-Regimes

Der sehr schnelle Rückgang der Gegenstimmen im Nationalsozialismus beruhte auf einer Mischung aus verstärkter sozialer Kontrolle, der Angst vor möglichen Repressalien, geglückter (nationalistischer) Wahlpropaganda, der im Laufe der Zeit zunehmenden massenhaften Zufriedenheit über den aufrüstungsbedingten Wirtschaftsaufschwung und der Integrationskraft der NS-Volksgemeinschaft.

Zwar erlauben die Wahlergebnisse aufgrund ihres Zustandekommens keine exakte Quantifizierung der Zustimmung im Nationalsozialismus, doch sind sie als Mindestmaß der artikulierbaren Ablehnung zu werten, die die Machthaber zulassen mussten, um bei einer „Wahl ohne Wahl“ noch als glaubwürdig angesehen zu werden. ${ }^{83}$

Otmar Jung hat vorgeschlagen, als Gradmesser des Dissenses sich ,nach dem aus der Rundfunktechnik bekannten Modell der Amplitudenmodulation" zu orientieren, d.h. die Gesamthöhe der Zustimmung als die Trägerfrequenz zu werten, auf der das Regime ,sendete“ und die davon am stärksten nach unten und oben abweichenden Ergebnisse zu analysieren: „gefragt wird mithin, wo einzelne Abstimmungswerte und um wieviel und warum von jenem Durchschnittswert abwichen "84 Der Verfasser folgt dieser Ansicht, da so selbst die fast 100prozentigen Ergebnisse von 1936 und 1938 in die Analyse einbezogen werden können. Die Anschaulichkeit dieses Modells soll am Beispiel der 35 Reichstagswahlkreise für die Jahre 1933 und 1934 aufgezeigt werden, wo sich folgendes Bild ergibt [Abb. 2 u. 3]:

83 Die folgenden statistischen Berechnungen beruhen auf: Statistik des Deutschen Reichs, Bd. 449, 1935. Bd. 497, 1936. Bd. 531, 1939. - Statistische Mitteilungen über den hamburgischen Staat, Nr. 32, S. 96, 1934. Aus Hamburgs Wirtschaft und Verwaltung, Nr. 6, S. 138, 1934.

84 Jung, Plebiszit (Fn. 28), S. 50. 


\section{Abweichungen der Ja-Stimmen vom Reichsdurchschnitt \\ (Mittelwert: VA Nov. 1933, RTW Nov. 1933, VA Aug. 1934)}

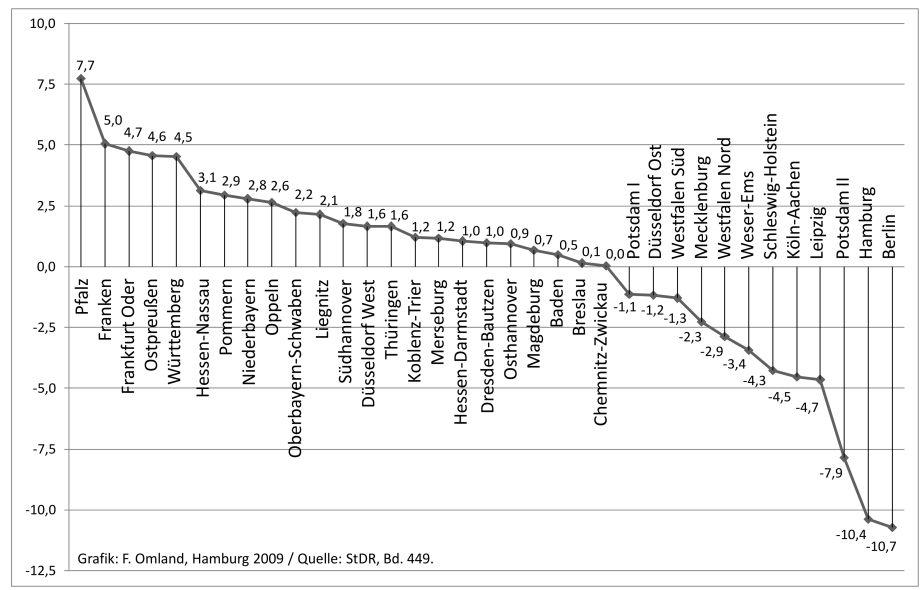

Lesehilfe:

Der Reichstagswahlkreis Pfalz wich bei allen drei Scheinwahlen durchschnittlich um 7.7 Prozentpunkte vom Zustimmungsergebnis im Deutschen Reich ab und war damit die Hochburg der Nationalsozialisten.

\section{Abweichungen der Ja-Stimmen vom Reichsdurchschnitt (Mittelwert: VA Nov. 1933, RTW Nov. 1933, VA Aug. 1934)}

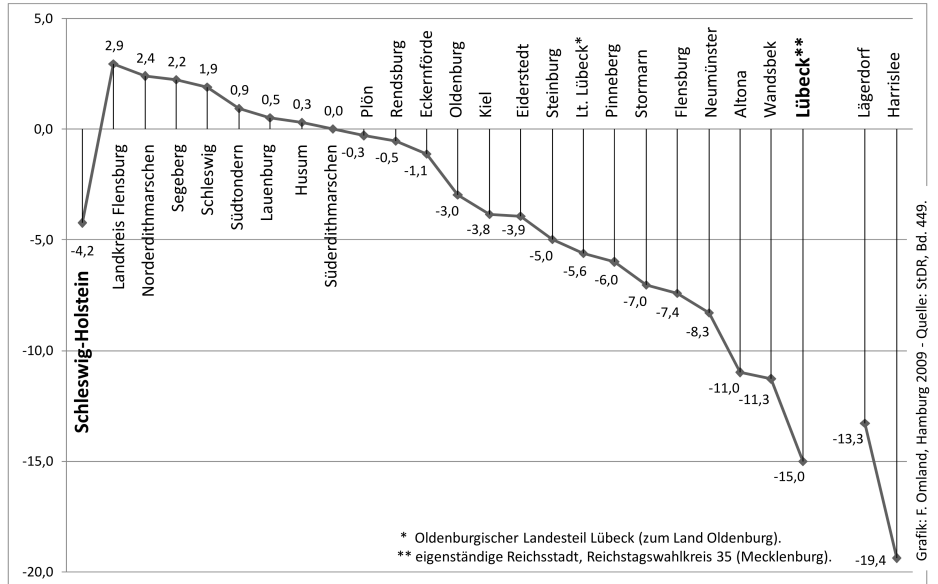

Lesehilfe:

Die Gemeinde Harrislee bei Flensburg wich bei allen drei Scheinwahlen durchschnittlich um 19,4 Prozentpunkte vom Zustimmungsergebnis im Deutschen Reich ab und war damit die Hochburg der Gegner im Nationalsozialismus in Schlesiwg-Holstein.

Danach führen die Wahlkreise Pfalz, Franken, Frankfurt/Oder, Ostpreußen und Württemberg reichsweit die Liste derjenigen an, die weit überdurchschnittlich für die Nationalsozialisten gestimmt haben. Dagegen finden sich auf der Gegenseite mit Berlin, Hamburg, Potsdam II, Leipzig und Köln-Aachen urban-industriell geprägte Regionen, die weit unter dem Reichsdurchschnitt lagen. ${ }^{85}$

85 Die Übertragung des Modells auf sämtliche Abstimmungen von 1933-1938 bedarf der Berücksichtigung der territorialen Veränderungen (Groß-Hamburg-Gesetz 1937, Eingliederung 
Folgt man Jungs Auswertungen der Ergebnisse auf Ebene der Wahlkreise, der Großstädte und einzelner Gemeinden, so gab es 1933 und 1934 ein Stadt-Landgefälle ${ }^{86}$. Er geht bei den Zustimmungsverlusten von 1933 auf 1934 davon aus, dass diese auf einen Anstieg der Ablehnung im katholischen Milieu zurück zu führen sind, während das Niveau der Gegenstimmung aus der politischen Arbeiterschaft erhalten blieb ${ }^{87}$ und auch Teile der bürgerlichen Wahlberechtigten für Nein-Stimmen verantwortlich sind. ${ }^{88} 1936$ konstatiert er, dass die ausdrückliche Opposition stark zurückgegangen und nur noch in neun Verwaltungsgebieten noch mindestens 4,0 Prozent Gegenstimmen zu verzeichnen waren, ${ }^{89}$ wobei er mangels reichsweiter statistischer Quellen die Wahlfälschung unberücksichtigt lässt. (Für Schleswig-Holstein weisen Indizien darauf hin, dass die Ablehnung in der Tendenz ähnlich hoch ausgefallen ist wie 1934). ${ }^{90}$ Für 1938 weist Jung reichsweit 14 Gemeinden mit einer Zustimmung unter 90 Prozent nach und belegt, dass im ländlichen Raum z.T. die JaStimmen noch darunter lagen. ${ }^{91}$ In vielen Punkten kann man diesen Analysen folgen, doch soll auf eine methodische Schwäche hingewiesen werden: „Als eindeutiger Nachweis für Sachopposition taugen also bei einer Volksabstimmung auch unter diktatorischen Bedingungen nur die abgegebenen gültigen Nein-Stimmen", so Jung. ${ }^{92}$ Doch leider befördert diese Methode Fehlschlüsse aufgrund der Nichtberücksichtigung der unterschiedlichen Wahlbeteiligung (siehe Exkurs). Ebenso hält der Verfasser den Argumenten Jungs über die Nichtwähler - diese sollen nicht generell als Opposition gewertet werden - entgegen, dass die „Abstimmung mit den Füßen“ in manchen Regionen eindeutig als Beleg für bewusstes resistentes Verhalten $\mathrm{zu}$ werten ist; dazu weiter unten mehr [Abb. 4]. ${ }^{93}$

des Saargebietes nach 1935, Änderungen in den Gebietsständen von Berlin, Potsdam I und II). Doch selbst bei den davon nicht betroffenen Wahlkreisen bleibt eine starke Kontinuität erhalten.

86 Jung, Plebiszit (Fn. 28), S. 50 ff. (1933), 71 (1934).

87 Jung, Plebiszit (Fn. 28), S. 69. - Hinweis: Erste Wählerwanderungsanalysen deuten an, dass das katholische Milieu nicht überdurchschnittlich an Nein-Stimmen beteiligt war.

88 Jung, Plebiszit (Fn. 28), S. 68. Als Beleg dienen ihm die Berliner bürgerlichen Bezirke Wilmersdorf und Charlottenburg. Wahlstatistisch ist das unstatthaft, wenn auch plausibel.

89 O. Jung, Wahlen und Abstimmungen im Dritten Reich 1933-1938, in: E. Jesse/K. Löw (Hrsg.), Wahlen in Deutschland, 1998, S. 85 f. - Aufgrund des von Jung gewählten Maßstabs (abgegebene Stimmen) kommt Omland, Du wählst (Fn. 10), S. 154, zu leicht abweichenden Ergebnissen. Der Verfasser kann für 24 Verwaltungsgebiete maximale Zustimmungen von $95 \%$ der Wahlberechtigten nachweisen. Das schwächste Ergebnis weist der Stadtkreis Wesermünde/ Wkr. 15 (Osthannover) auf: 10,5 \% Wahlenthaltungen, 86,8 \% Zustimmung der Wahlberechtigten.

90 Omland, Du wählst (Fn. 10), S. 156.

91 Jung, Plebiszit (Fn. 28), S. 120; ebd. Fn. 60. - Omland, Du wählst (Fn. 10), S. 182 f., belegt, dass von 120 Gebietseinheiten Schleswig-Holsteins lediglich in neun Gemeinden die Zustimmung unter 95 \% lag. Die stärkste Kontinuität im Zeitverlauf 1933-1938 wiesen die ehemalige KPD-Hochburg Lägerdorf (3.000 Einw.) und das durch die dänische Minderheit geprägte Harrislee (2.000 Einw.) auf.

92 Jung, Plebiszit (Fn. 28), S. 49.

93 Jung, Plebiszit (Fn. 28), S. 48. 


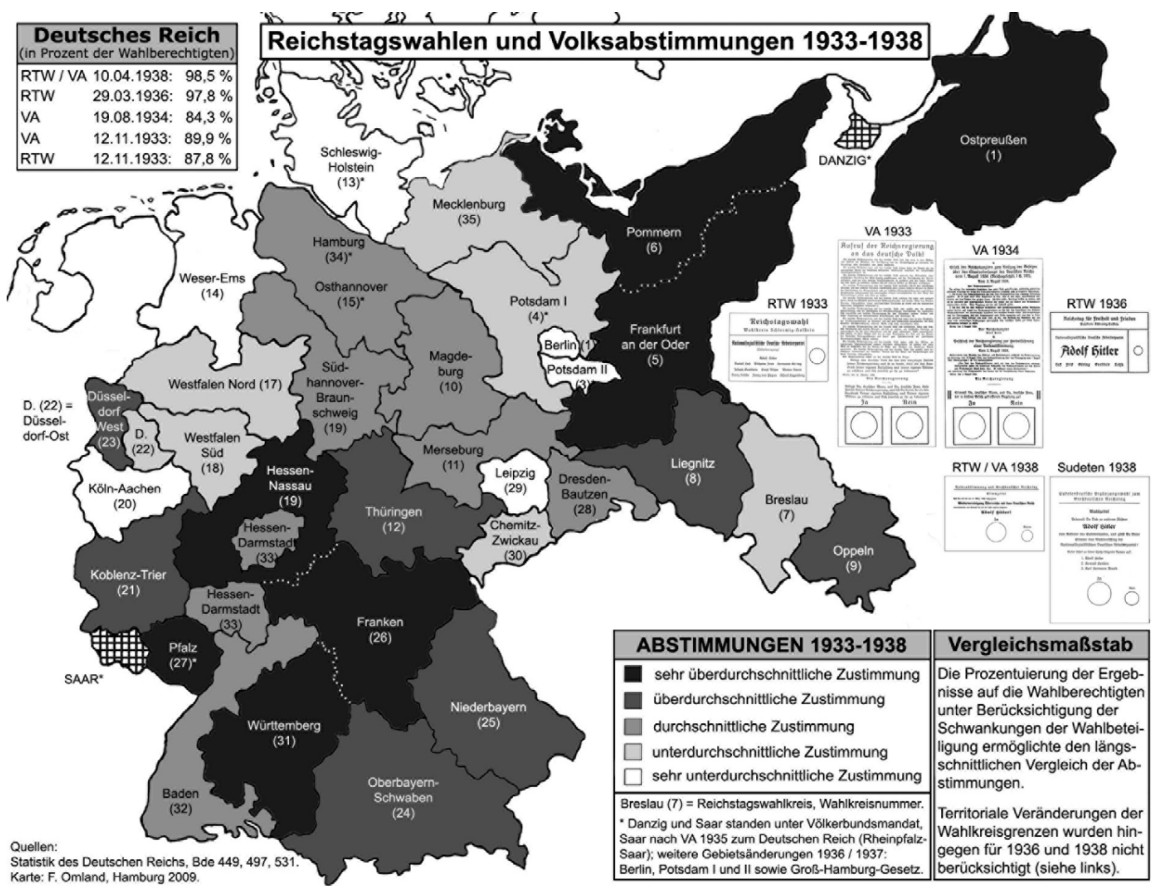

Auf regionaler Ebene blieb Schleswig-Holstein über den gesamten Zeitverlauf von 1933 bis 1938 immer einer derjenigen Reichstagswahlkreise mit relativ schwachen Resultaten für das NS-Regime. ${ }^{94}$ Er rangierte im November 1933 an fünfter bzw. vierter Stelle, im August 1934 an siebter, im März 1936 an zweiter und 1938 an siebter Stelle von allen Wahlkreisen. ${ }^{95}$ Nach den Großstädten Hamburg, Berlin und der Region Potsdam II, die reichsweit kontinuierlich führend in der Ablehnung/Opposition waren, dürfte der Wahlkreis 13 damit eine der „Hochburgen“ derjenigen gewesen sein, die überdurchschnittlich oft mit Nein gestimmt haben. Tatsächlich unterschied sich das Stimmverhalten in Schleswig-Holstein im Kern nicht von dem in anderen Regionen mit hoher Oppositionsquote, denn auch hier beruhten die NeinStimmen auf dem Stimmverhalten der ehemaligen KPD- und SPD-Anhänger. So resultierten im November 1933 die stärksten Abweichungen zuungunsten der

94 Sämtliche Ergebnisse auf Ebene der Kreisgebiete sind dokumentiert in einer wahlstatistischen Datenbank des Verfassers auf www.akens.org/akens/texte/diverses/wahldaten/index.html (Zugriff: 26.7.2009).

95 RTW 1933: Berlin (77,4), Hamburg (78,1), Leipzig (82,7), Potsdam II (79,5), Schleswig-Holstein (84,1). VA 1933: Hamburg (80,1), Berlin $(80,2)$, Potsdam II $(84,0)$, Schleswig-Holstein $(84,9)$. VA 1934: Hamburg (72,6), Köln-Aachen $(75,9)$, Berlin $(72,1)$, Westfalen Nord $(77,6)$, Leipzig $(80,3)$, Potsdam II $(74,9)$, Weser-Ems $(79,3)$ und Schleswig-Holstein $(80,3)$. RTW 1936: Hamburg (94,0), Schleswig-Holstein (95,7). RTW/VA 1938: Leipzig (95,2), Hamburg $(96,3)$, Weser-Ems $(96,9)$, Dresden-Bautzen $(97,0)$, Osthannover $(97,4)$, Chemnitz-Zwickau $(97,5)$, Schleswig-Holstein $(97,7$; alle Angaben in Prozent). 
Machthaber aus dem Wahlverhalten in den beiden Großstädten Altona und (schwächer) Kiel sowie den industriell geprägten Gemeinden im Umland Hamburgs und Lübecks: Je größer die Stadt, desto überdurchschnittlicher fielen die Wahlenthaltungen und Nein-Stimmen aus und umgekehrt galt, je kleiner die Gemeinde, desto höher fiel die Zustimmung aus. Ebenfalls als Dissens ist die Nichtabgabe des Reichstagsstimmzettels 1933 zu werten, wobei schätzungsweise 14.800 Wahlberechtigte - vornehmlich in urbanen Regionen ${ }^{96}$ - davon Gebrauch machten und in einzelnen Wahllokalen sogar bis zu 28,5 Prozent der Wähler so handelte. ${ }^{97}$ Bei den Volksabstimmungen stellte die Abgabe eines ungültigen Stimmzettels eine weitere Möglichkeit dar, seine generelle Ablehnung gegenüber dem Verfahren an sich zu bekunden..$^{98}$ Als ein Beleg kann das Wahlverhalten im Landkreis Eiderstedt (14.000 Einwohner) gewertet werden, der traditionellerweise zu den Regionen mit der geringsten Wahlbeteiligung gehörte. ${ }^{99}$ Angesichts der Perfektionierung der Wahlschleppdienste konnten sich die ins Wahllokal gezwungenen Menschen dem Verfahren zwar nicht mehr entziehen, doch drückten sie hier ihren generellen Unmut darüber auch durch eine ungültige Stimme aus. ${ }^{100}$ Diese These wird durch die Ergebnisse der Wählerwanderungsanalysen für Berufsgruppen gestützt (s.u.), denn hier zeigt sich, dass die erwerbstätigen „Angestellten“ und „Arbeiter“ überdurchschnittlich oft ungültige Stimmzettel abgaben. ${ }^{101}$ Diese Berufsgruppen entschieden sich also auch bei Volksabstimmungen bewusst dafür, sich dem Verfahren an sich zu entziehen.

Starke Kontinuitäten in den Jahren 1933 bis 1938 lassen sich mit dem überlieferten statistischen Material bei den Wahlenthaltungen und den Nein-Stimmen in

96 Omland, Du wählst (Fn. 10), S. 64 f. und 72 f. Von allen nicht abgegeben Stimmzetteln stammten $41 \%$ aus den beiden Großstädten. - Die Gemeinde mit der höchsten Quote stellte Kronshagen bei Kiel dar (2.461 Einwohner): Wahlenthaltung 13,8 \%/Nichtabgabe 8,1 \%.Vgl. dazu Jung, Plebiszit (Fn. 28), S. 52 ff.

97 Vgl. Omland, Du wählst (Fn. 10), S. 73. - Stichproben für Kiel (170 Wahllokale), Lübeck (105) und Neumünster (30) ergaben Stimmzettelrückgänge von 28,5\% (Lübeck), 18,7 \% (Kiel) und 12,4\% (Neumünster) in einzelnen Wahllokalen.

$9834 \%$ aller ungültigen Stimmen (VA) stammten aus den Großstädten, nur $22 \%$ aus den ländlichen Regionen unter 2.000 Einwohnern, vgl. Omland, Du wählst (Fn. 10), S. 64 f.

991938 lag die Wahlbeteiligung mit 98,2\% nach Flensburg mit 97,5\% an der zweiter Stelle der Enthaltungen, die Zustimmung fiel am schlechtesten aus (95,5\%), ebenso die ungültigen Stimmen $(0,8 \%)$ im Vergleich zu den anderen Kreisen.

100 Vgl. Omland, Du wählst (Fn. 10), S. 182, Tab. 2. 1938. Fast die Hälfte aller ungültigen Stimmen wurden in den ländlichen Regionen mit unter 2.000 Einwohnern abgegeben, während dies 1933 nur $22 \%$ (VA) bzw. $31 \%$ (RTW) waren.

101 Verteilung der Berufsgruppen unter den Wahlberechtigten: Selbständige 8,7\%/Beamte 5,5\% /Angestellte 10,2\%/Arbeiter 13,7\%/erwerbslose Arbeiter 12,2\%/erwerbslose Angestellte 3,0\%/Mantelbevölkerung 46,7\% (in der Regel Hausfrauen und Berufslose sowie Rentner). Anteil der Angestellten/Arbeiter an allen ungültigen Stimmen 1933: 11,7/50,8 \%; 1934: $39,8 \% / 44,2 \%$. 
den größeren Städten des Wahlkreises aufzeigen: ${ }^{102}$ so in Altona, ${ }^{103}$ Neumünster ${ }^{104}$ und Wandsbek - alles ehemaligen Hochburgen der verbotenen Arbeiterparteien - sowie der Hochburg der dänischen Minderheit, Harrislee bei Flensburg, ${ }^{105}$ und der ehemals kommunistischen Hochburg Lägerdorf/Kreis Steinburg. Bezieht man die bisherigen Auswertungen für bestimmte Arbeiterstadtteile Hamburgs und das bis 1937 eigenständige Lübeck ein, belegen alle statistischen Analysen eindrucksvoll den Zusammenhang zwischen ehemaligen Hochburgen der KPD und SPD und einer hohen Oppositionsquote im Nationalsozialismus. ${ }^{106}$ So verbuchte Lübeck 1933 die reichsweit schlechtesten Ergebnisse für die Machthaber (71,6 resp. 71,9 Prozent) und sollte auch 1934 (73,5 Prozent) in der Spitzengruppe der Ablehnung zu finden sein. ${ }^{107}$ Der Hamburger Stadtteil Langenhorn gehörte 1933 (58,8 bzw. 61,2 Prozent) und 1934 (57,0 Prozent) reichsweit ebenso zur Spitzengruppe mit den geringsten Ja-Stimmen. Und für einzelne Wahllokale fiel diese Quote sogar bis auf unter 50 Prozent ab. ${ }^{108}$

Unter Einbeziehung der Reichstagswahl vom März 1936 lässt sich nach bisherigen Auswertungen von zweierlei ausgehen: erstens war es in Stadtkreisen und Großstädten einfacher möglich, mit Nein zu stimmen. Zweitens hält sich im Zeitverlauf von 1933 bis 1938 in einzelnen Gemeinden hartnäckig eine - wenn auch immer kleinere werdende - Anzahl von Wahlberechtigten, die mit Nein stimmten. ${ }^{109}$

102 Altona, Neumünster und Wandsbek gehörten 1936 zu den zehn Kreisen (von 104 reichsweit) in denen es die stärkste Opposition gegen das Regime gegeben hatte. Lägerdorf hatte noch 1938 das höchste Nein-Stimmenergebnis zu verzeichnen (4,9\%), Harrislee die höchste Wahlenthaltung (2,8\%) und gehörte mit 3,4\% zu den zehn Gemeinden mit der geringsten Zustimmung. Vgl. Omland, Du wählst (Fn. 10), S. 154, 156 sowie 182.

103 Vgl. Omland, Altona (Fn. 10), S. 57 ff.

104 Vgl. Omland, Neumünster (Fn. 79).

105 RTW 1933/VA 1933/VA 1934: Harrislee 13,3\%/13,3 \%/14,4 \% (RV 1938: 2,8 \%; zweithöchste Wahlenthaltung in Schleswig-Holstein).

106 Der Stadtteil Hamburg-Langenhorn (7.320 Einw.) wies kontinuierlich eine extrem niedrige Wahlbeteiligung und hohe Ablehnungsquoten auf: RTW 1933/VA 1933/VA 1934: 25,9\%/ 22,8 \%/22,5 \%. Vgl. zu Details Omland, Du wählst (Fn. 10), S. 68 f., 114, 182. - Zur Situation in Hamburg vgl. F. Bajohr, Die Zustimmungsdiktatur, in: Forschungsstelle für Zeitgeschichte (Hrsg.), Hamburg im „Dritten Reich“, 2005, S. 94 und S. 99.

107 Vgl. Jung, Plebiszit (Fn. 28), S. 53. Vgl. Omland, Lübeck (Fn. 72), S. 152, 162 ff. - Eine Zusammenstellung nach Gauen der NSDAP weist Lübeck das schlechteste Endergebnis in Prozent der abgegebenen gültigen Stimmen zu: Bundesarchiv Berlin, NS 1501/5355. Darin: „Die Volksabstimmungen am 12. November 1933 und 19. August 1934“, o.O., o.D., ohne Autoren und Verlagsnennung.

108 Hamburg-Barmbek, Wahllokal 370/7: RTW 1933: 46,4 \%, VA 1933: 49,3 \% der Wahlberechtigten.

109 Vgl. dazu Omland, Du wählst (Fn. 10), S. 154 ff. - Jung, Wahlen (Fn. 89), S. 86. 


\section{Die politische und soziale Zusammensetzung der Wählerschaft}

Die Methoden der modernen Wahlforschung (Logit-Modell von Sören Thomsen ${ }^{110}$ ) ermöglichen für 1933 und 1934, Schätzungen zu den Wählerwanderungen anzustellen. Für Schleswig-Holstein, ${ }^{11}$ die Stadt Neumünster ${ }^{12}$ und die sächsische Stadt Chemnitz ${ }^{113}$ liegen deshalb qualitative Aussagen zum Wählerverhalten vor. In der Tendenz bestätigen alle Ergebnisse für die Doppelabstimmung von November 1933 sowie die Volksabstimmung 1934, dass die Wahlberechtigten bewusst zwischen außenpolitischen und innenpolitischen Fragestellungen unterschieden. Für die Wählerwanderungen in Schleswig-Holstein zeigt sich, dass diejenigen, die bei der Reichstagswahl im März 1933 die NSDAP oder die DNVP ${ }^{114}$ gewählt hatten, zu 96 bis 98 Prozent auch im November 1933 und zu 94 Prozent im August 1934 für das Regime stimmten. Dagegen gab lediglich etwas mehr als jeder dritte ehemalige KPD-Wähler von März 1933 im November 1933 den Nationalsozialisten seine Zustimmung, während es seitens der ehemaligen SPD-Wähler immerhin 72-74 Prozent waren. ${ }^{15}$ Diese Tendenzen verstärkten sich bei der Volksabstimmung im August 1934, so dass nur noch zwei von drei SPD-Wählern für Hitler votierten und auch von der KPD-Anhängerschaft noch einmal etwas weniger für das NS-Regime stimmten. ${ }^{16}$ Die Tatsache, dass von 104 auswertbaren Gemeinden und ländlichen Restbezirken Schleswig-Holsteins bei der Volksabstimmung 1934 lediglich 33 über dem Reichsdurchschnitt der Ja-Stimmen für Hitler lagen, belegt eindrucksvoll, dass die Opposition gegen die Vereinigung von Reichspräsidenten- und Reichskanzleramt flächendeckend ausfiel [Abb. 5]. ${ }^{117}$

110 Thomsen, Elections (Fn. 78). - Zur Anwendung siehe D. Hänisch, Die österreichischen NSDAP-Wähler. Eine empirische Analyse ihrer politischen Herkunft und ihres Sozialprofils, 1998 [Der Verfasser kooperiert seit mehreren Jahren sehr konstruktiv mit Dr. Dirk Hänisch, Bonn, dem er auch die Berechnungen nach dem Logit-Modell verdankt].

111 Schleswig-Holstein: 104 Gemeinden sowie ländliche Restbezirke unter 2.000 Einw. - Vgl. Omland, Du wählst (Fn. 10), S. 73 ff. und S. 115 ff.

112 Neumünster: 28 Wahllokale. - Omland, Neumünster (Fn. 79), S. 35 ff. und Omland, Du wählst (Fn. 10), S. 199.

113 Chemnitz: 200 Wahllokale. - Vgl. D. Hänisch, Die Wahl- und Abstimmungsergebnisse in Chemnitz 1933 und 1934, in: Stadtarchiv Chemnitz (Hrsg.), Chemnitz in der NS-Zeit, Beiträge zur Stadtgeschichte 1933-1945, 2008, S. 30 f.

114 Im März 1933 war die DNVP zusammen mit dem Stahlhelm als Kampffront Schwarz-WeißRot angetreten.

115 Wählerwanderungen RTW März 1933 zu RTW/VA November 1933: NSDAP (98 \%/97\%), DNVP (96\%/97\%), Nichtwähler (85\%/84 \%), Sonstige (DVP, DStP, Andere; $66 \% / 75 \%$ ), SPD (72\%/4\%), KPD (38\%/38\%). - Details siehe Omland, Du wählst (Fn. 10), S. 75-77.

116 Wählerwanderungen RTW März 1933 zu VA 1934: NSDAP $94 \%$; DNVP 94 \%; SPD $67 \%$; KPD 34 \%. Details siehe Omland, Du wählst (Fn. 10), S. 115 f.

117 Omland, Du wählst (Fn. 10), S. 112 ff. 
Geschätzte Wählerwanderungen in Schleswig-Holstein:

Reichstagswahl 5. März 1933 zu Volksabstimmung 19. August 1934

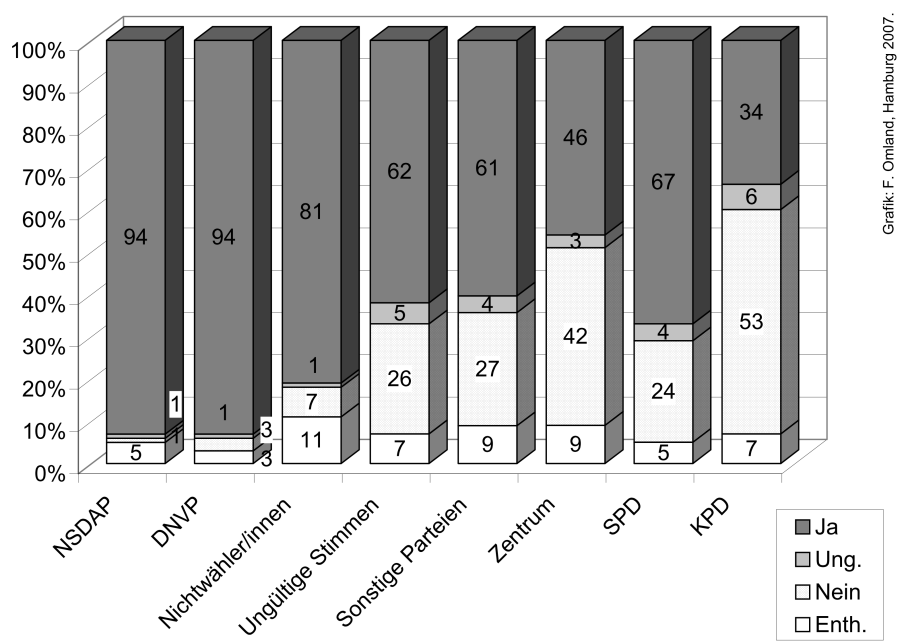

Lesehilfe:

Von allen Wählern, die am 5. März 1933 einen ungültigen Stimmzettel abgegeben haben, stimmten 62 Prozent bei Volksabstimmung am 19. August 1934 mit Ja.

Von allen Wählern, die am 5. März 1933 der KPD ihre Stimme gegeben hatten, votierten bei der Volksabstimmung im August 1934 insgesamt 53 Prozent mit Nein.

Ähnlich sieht es im November 1933 für die 40.000 Einwohner zählende Stadt Neumünster aus, wo vier von zehn ehemaligen KPD-Wählern sich für die NSDAP entschieden und fast die Hälfte von ihnen den Völkerbundsaustritt befürwortete. Und für die Großstadt Chemnitz in Sachsen lässt sich bei allen drei Scheinwahlen für die vier Hauptprotagonisten von März 1933 (SPD, KPD, NSDAP, DNVP) nachweisen, dass der Völkerbundaustritt weniger abgelehnt wurde als die NSDAP-Einheitsliste 1933 und Hitlers Übernahme des Reichspräsidentenamtes 1934. Am stärksten zeigte sich dies 1934, wo noch 23 Prozent aller ehemaligen SPD-Wähler vom März 1933 gegen Hitler stimmten, während im November 1933 lediglich 14 Prozent gegen den Austritt aus dem Völkerbund votierten. Damit belegen die Ergebnisse aus allen drei Untersuchungsgebieten, dass sich die Nein- und ungültigen Stimmen quantitativ den ehemaligen Anhängern der verbotenen Arbeiterparteien zuordnen lassen und in der Tendenz entweder die ehemalige KPD-Wählerschaft die größte Oppositionsgruppe gegen den Nationalsozialismus bildete - wie für Schleswig-Holstein und Neumünster - oder die SPD - wie für Chemnitz belegbar. Insgesamt stellte die ehemalige Anhängerschaft der beiden verbotenen Arbeiterparteien in diesen drei Gebieten zwischen 74 und 92 Prozent all derjenigen, die gegen die Nationalsozialisten votiert hatten. ${ }^{118}$

Diese Ergebnisse werden durch die Ermittlung des Wahlverhaltens der Berufsgruppen, ausdifferenziert nach den drei Wirtschaftssektoren (Agrar-/Industrie und Handwerk-/Dienstleistungen und Handelssektor) sowie nach agrarisch- und urbangeprägten Regionen, in Schleswig-Holstein untermauert. Aus Platzgründen sollen 
die recht eindeutigen Ergebnisse hier nur summarisch dargestellt werden: ${ }^{119}$ Je höher der Anteil am Agrarsektor in den Gemeinden ausfiel, desto höher war die Zustimmung, während es sich für den Bereich „Industrie und Handwerk“ bzw. „Dienstleistungen und Handel" umgekehrt darstellte. In letzteren lag die Zustimmung um mindestens sieben bis neun Prozentpunkte unter denen im Agrarsektor und sank 1934 auf knapp 76 Prozent ab. ${ }^{120}$ Bei der Auswertung nach Berufsgruppen ${ }^{121}$ zeigten die erwerbslosen sowie die erwerbstätigen „Arbeiter“ die überdurchschnittlichste Ablehnung gegen das NS-Regime. In den ländlichen Gebieten waren sie 1933 sogar fast die einzige nennenswerte Opposition, denn sie stellten hier quantitativ zwischen 69 und 97 Prozent aller Gegenstimmen zum NS-Regime, ${ }^{122}$ in der Regel gefolgt wenn auch auf schwächerem Niveau - von den (erwerbslosen) „Angestellten“. ${ }^{123}$

Die Wahlanalyse macht deutlich, dass es insbesondere das ehemalige kommunistische Wählermilieu sowie (wenn auch schwächer) das sozialdemokratisch geprägte Milieu in der verbotenen politischen Arbeiterbewegung war, welches die Möglichkeiten der Regimegegner mit „Nein“ zu stimmen, erhöhte. Anhänger der KPD, deutlich schwächer der SPD, die sehr kleine katholische Minderheit ${ }^{124}$ und regionalspezifisch die dänische Minderheit stellten das Gros der Gegenstimmen und Wahlenthaltungen in Schleswig-Holstein. Dabei griffen die Wahlberechtigten auf alle Möglichkeiten zurück: Sie boykottierten die Scheinwahlen, stimmten mit „Nein“ oder machten den Stimmzettel ungültig.

\section{Verhaltensoptionen der Wahlberechtigten}

Die Wahlberechtigten, die bei den Abstimmungen im Nationalsozialismus mit „Nein“ stimmten, setzten sich einem hohen persönlichen Risiko aus. Sie handelten dabei politisch, öffentlich und gegen das NS-Regime gerichtet, was mindestens als

119 Vgl. Omland, Du wählst (Fn. 10), S. 79 ff. (1933), 118 ff. (1934).

120 Zustimmung der Wahlberechtigten in den Gemeinden mit den höchsten Anteilen an Erwerbstätigen im Agrarsektor: RTW 1933: 89,2 \%, VA 1933: 89,8 \%, VA 1934: 85,3 \%. Zustimmung in den Gemeinden mit den höchsten Anteilen am Sektor „Industrie“: 80,2 \%/ $79,6 \% / 76,3 \%$ und im Dienstleistungssektor $81,6 \% / 82,1 \% / 76,8 \%$.

121 Zur sozialen Schichtung Schleswig-Holsteins und zu methodischen Überlegungen siehe Omland, Du wählst (Fn. 10), S. 28 ff.

122 Vgl. dazu Omland, Du wählst (Fn. 10), S. 80 ff. und die Tabelle 7 b, S. 204.

123 Zustimmung in Prozent der Wahlberechtigten, urbane Regionen, RTW 1933/VA 1933/VA 1934: Arbeiter 67,9/71,8/72,4; erwerbslose Arbeiter 65,6/80,4/79,6; Zustimmung, ländliche Regionen: Arbeiter 71,8/84,4/61,2; erwerbslose Arbeiter 80,4/50,9/60,1. - Die geringste Zustimmung zeigten sich unter den Angestellten bei der RTW 1933 in den ländlichen Regionen mit 66,5 \%. - Zu Details siehe Omland, Du wählst (Fn. 10), S. 80 ff., 118 ff.

124 Das protestantische Schleswig-Holstein war „Diaspora“ des Zentrums gewesen. Eine Übertragbarkeit des Wahlverhaltens der ehemaligen Anhänger auf die Hochburgengebiete der Partei ist deswegen nicht statthaft. 
Dissens mit diesem zu werten ist. ${ }^{125}$ Das mögliche Verhaltensspektrum der Wahlberechtigten in Schleswig-Holstein war 1933 und 1934 noch so groß, dass die Mutigen unter ihnen bei jeder Abstimmung ihre Ablehnung artikulieren konnten, wenn sie denn bereit waren, mögliche negative Konsequenzen zu tragen.

Im Gegensatz zu Wahlen in der Demokratie waren die Abstimmungen in der Diktatur weder frei noch pluralistisch, und die Wahlberechtigten konnten sich nicht sicher sein, dass das Wahlgeheimnis gewahrt blieb. Die Entscheidung, trotzdem gegen den erklärten Willen des NS-Regimes zu handeln, sollte deshalb nicht gering geschätzt werden. Es war eine bewusste, gegen die Machthaber gerichtete Handlung, die einigen persönlichen Mut erforderte. Dieser so geäußerte Dissens mit dem NSRegime blieb gleichwohl ein Minderheitenphänomen, und die von den Nationalsozialisten angestrebte „Volksgemeinschaft“ war mit diesen Wahlberechtigten nicht zu erreichen. Die Ideologie von der „Volksgemeinschaft“ zielte auf ein „Deutschland total nationalsozialistisch" 126 ab und war in der Wirklichkeit bei jedem Urnengang zum Scheitern verurteilt, da jede noch so geringe Abweichung durch Partei und Regierung als grundsätzliche Infragestellung des Regimes betrachtet werden musste. Nach der NS-Ideologie hätten alle „Volksgenossen“ freiwillig für das Regime stimmen müssen, was letztendlich angesichts der gesellschaftlichen Realität völlig absurd war. Dementsprechend scheiterte das NS-Regime am eigenen Verfahren, denn durch die Abhaltung von Reichstagswahlen und Volksabstimmungen musste es zu jedem Zeitpunkt mit Gegenstimmen rechnen. Die aber durfte es nicht geben.

In der NS-Diktatur erforderte es angesichts der Ungewissheit darüber, ob Gegenstimmen zurückverfolgt und das Wahlgeheimnis gebrochen werden würde, persönlichen Mut, im Wahllokal mit „Nein“ zu stimmen oder einen ungültigen Stimmzettel abzugeben. Das Fehlen dieses Mutes ist aber nicht zwangsläufig als Zustimmung zum NS-Regime zu werten. Gegenstimmen setzten ein deutliches Zeichen und wurden - wie die Propaganda der Nationalsozialisten zeigte - auch genauso wahrgenommen. Ebenso brauchte es persönlichen Mut, trotz des „Wahlschleppdienstes" der SA gar nicht zur Wahl zu gehen bzw. sich durch die Ausstellen eines Stimmscheins der sozialen Kontrolle vor Ort zu entziehen.

Organisierter Widerstand gegen die Abstimmungen in der NS-Diktatur fand fast ausschließlich durch die illegale KPD und schwächer die SPD statt. Seitens der dänischen Minderheit und der Zeugen Jehovas gab es Wahlenthaltungsaufrufe, und auch die Anhänger des antisemitischen Tannenbergbundes äußerten bei den Abstimmungen ihren Unmut. Hinzu zu zählen ist auf individueller Ebene eine Reihe von einzelnen Wahlberechtigten, die die Abstimmungen dazu nutzten, ihre Ablehnung des Regimes zu artikulieren, ohne dass dies auf die Zugehörigkeit zu einer ganz bestimmten (politischen) Gruppe zurückzuführen wäre.

125 Vgl. zur Begriffsbildung E. Imberger, Widerstand ,von unten“. Widerstand und Dissens aus den Reihen der Arbeiterbewegung und der Zeugen Jehovas in Lübeck und Schleswig-Holstein 1933-1945, 1991, S. 26 f.

126 Kieler Zeitung 13.11.1933. 


\section{3. „Wahlen ohne Wahl“ im NS-Staat als Beleg für die „Zustimmungsdiktatur"}

Das Verhalten der Wahlberechtigten ist als ein Indiz für die Zustimmungsdiktatur $^{127}$ (im Sinne einer „Zustimmung zum Erfolg“ der NS-Diktatur) zu werten und spiegelt den milieuübergreifenden nationalen Grundkonsens wider. ${ }^{128}$ Dieser ermöglichte es den Nationalsozialisten vor dem Hintergrund von anfangs schwierigen wirtschaftlichen Rahmenbedingungen, selbst in den ehemaligen linken Hochburgen die Mehrheit der Wahlberechtigten für sich zu gewinnen oder sie zumindest davon abzuhalten, gegen sie zu stimmen.

Umgekehrt zeigen die Wahlanalyse auf Basis der Ergebnisse in den Wahllokalen und den Gemeinden sowie die Auswertung der schriftlichen Quellen für SchleswigHolstein und sein Umland: In den Regionen, wo der Verfolgungsapparat aus Gestapo und Polizei gemessen an anderen Städten noch relativ schwach war, die NSDAP mit einer starken SPD und KPD-Minderheit zu kämpfen hatte und der illegale Widerstand in der Stadt relativ stark organisiert war, konnten die Wahlberechtigten am ehesten ihre Ablehnung am Wahltag ausdrücken. Gefestigte Ideologien, soziale Netzwerke und das Vorhandensein eines Gegenmilieus beförderten also potentiell die Ablehnung bei den Abstimmungen.

\section{Was bleibt zu tun?}

Zukünftig bieten sich zwei Ansätze für weitere Forschungen an: Zum einen können die Ergebnisse für Schleswig-Holstein mit denen aus anderen strukturell ähnlichen Regionen verglichen bzw. reichsweit flächendeckend kleinräumliche Daten analysiert werden. Zum anderen könnte die so genannte „Überwinterungs-These“ überprüft werden. Das ist die Frage, ob sich die Einstellungen (gerade im ehemaligen SPD- und KPD-Milieu) über den Nationalsozialismus hinaus erhalten haben. Konnten diese in der Diktatur ,überwintern“ und sich also auch nach 12 Jahren als verfestigte Sozialmilieus herausstellen? Dazu benötigen wir ein Untersuchungsgebiet, in dem die Wahlergebnisse von 1932 bis 1938 sowie der ersten demokratischen Nachkriegswahlen erhalten sind, und (!) es nur zu wenigen Bevölkerungsverschiebungen gekommen ist. Wenn sich hier starke Kontinuitäten im Wahlverhalten zeigen würden, wäre dies ein Indiz für die „Überwinterung“ von Einstellungen im Nationalsozialismus.

Für Schleswig-Holstein dürfte das Auffinden eines solchen Gebietes aufgrund der großen Zuwanderung von Flüchtlingen und Vertriebenen nach 1945 sowie der schlechten Wahlergebnis-Überlieferung leider nur schwer bis unmöglich zu reali-

127 G. Aly, Rasse und Klasse. Nachforschungen zum deutschen Wesen, 2003, S. 76 u. 246; ders., Hitlers Volksstaat. Raub, Rassenkrieg und nationaler Sozialismus, 2005, S. 36. - Für Hamburg siehe Bajohr, Zustimmungsdiktatur (Fn. 106), S. 69 ff., 121.

128 Ein mögliches indirektes Indiz stellen die Wahlergebnisse außerhalb des deutschen Reiches dar, so die Saarabstimmung 1935, die Landtagswahlen im Memelgebiet 1935 und die Wahlen in Danzig 1935. Vgl. dazu Falter u.a., Wahlen (Fn. 15), S. 114 ff. 


\section{Frank Omland}

sieren sein, so dass sehr wahrscheinlich andere Gebiete für die Forschung erschlossen werden müssten. 
